Research Article

\title{
Effect of Parameters on the Design of a Suspension System with Four Oblique Springs
}

\author{
Gianluca Gatti ii \\ Department of Mechanical, Energy and Management Engineering, University of Calabria, Rende 87036, Italy \\ Correspondence should be addressed to Gianluca Gatti; gianluca.gatti@unical.it
}

Received 20 January 2021; Revised 14 May 2021; Accepted 27 May 2021; Published 28 June 2021

Academic Editor: Jean-Jacques Sinou

Copyright (c) 2021 Gianluca Gatti. This is an open access article distributed under the Creative Commons Attribution License, which permits unrestricted use, distribution, and reproduction in any medium, provided the original work is properly cited.

\begin{abstract}
This paper presents the fundamental static and dynamic characteristics of a suspension system consisting of four linear springs arranged in an $X$-shaped configuration to achieve geometric nonlinearity. The particular interest is towards the design of a softening spring geometry realizing a quasi-zero stiffness behaviour at large deflections, and the influence of the system parameters is investigated. The static performance is studied in terms of the force-deflection curve and the dynamic performance in terms of the frequency response curve. The softening-hardening behaviour of the suspension leads to a frequency response which bends to the lower frequencies reaching a well-defined minimum. It is found that both the static and dynamic behaviours may be described in terms of a single parameter, and a simple closed-form expression is determined which links the damping in the system to the excitation amplitude to achieve the lowest possible resonance frequency.
\end{abstract}

\section{Introduction}

The ever growing demand to enhance the performance of mechanical systems and structures has recently pushed research efforts towards the exploitation of nonlinear effects rather than to their avoidance $[1,2]$. New solutions and innovative designs have been investigated recently to this purpose, especially in the field of nonlinear dynamics and vibration control [3,4]. Mathematical approaches have been adopted to investigate the dynamic behaviour of nonlinear oscillators, with specific emphasis to prescribed nonlinear functions of stiffness $[5,6]$ and damping $[7,8]$.

Practical applications of the benefits that nonlinearity can introduce into a mechanical system are reported in the field of energy harvesting from vibrations [9], vibration absorbers [10], shock isolators [11], vibration isolators [12], and elastic systems for potential energy increase [13]. In some cases, a nonlinear stiffness element with a quasi-zero stiffness (QZS) characteristic $[2,6,8,12,13]$ has been proposed to cope with the competing requirements of achieving a high-static stiffness to limit the static deflection and a low-dynamic stiffness to improve the dynamic performance. A cubic stiffness characteristic with hardening behaviour has been commonly reported, and a practical mechanical realization consists of a pair of linear springs located perpendicularly to the direction of motion, which incline as the oscillator moves [2]. A global nonlinear behaviour of hardening type assures stability at the equilibrium configuration, where the QZS effect is often required, and this characteristic is obtained by combining elastic elements with positive and negative stiffness in parallel. The exclusive use of a dominant softening stiffness effect, which can be also practically obtained by using magnets arranged in an attractive configuration [14], has the potential disadvantage to eventually lead to a bistable or snap-through behaviour $[2,15]$ for large displacements or even instabilities, which could be undesired or detrimental in some cases.

In this paper, a softening-hardening behaviour is achieved by simply arranging linear springs in an $X$-shaped mechanical configuration, realizing a quasi-zero stiffness effect for large deflections. Following the preliminary idea presented in [16], a series of research works have recently addressed its exploitation to develop innovative vibration isolators [17-19], and it is believed that such characteristic may be of interest to other applications, so that it is further studied in this paper. 
Respect to the short communication presented in [16], where the system was excited by a force on the oscillating mass and no effect of gravity was considered, this paper presents a more attractive engineering configuration where the system is base-excited and the effect of the gravity on the static equilibrium is taken into account. This in turn involves the introduction of more design parameters which may be exploited for a better tuning to the desired application. The contribution of the present paper is thus a step forward towards the practical design of the system. A deeper theoretical insight is undertaken which shed new light on the key parameters of the system. A simple and interesting relation between the excitation amplitude and the system damping is uncovered from the study of the frequency response. The analytical and numerical analysis presented in this paper allows a fundamental understanding of the nonlinear characteristics of the system, underpinning prospective pioneering applications which can be potentially attractive to the engineering community for vibration control issues at large [20].

\section{Force-Deflection Characteristic}

The model of the system considered in this work is illustrated in Figure 1(a), and it consists of a mass $m$ suspended by two pairs of linear springs arranged in an oblique geometric configuration inside a casing. The pair of springs at the top (bottom) have stiffness $k_{1}\left(k_{2}\right)$, and the geometry of the spring arrangement is defined by the dimension $a$ and $b$, as indicated in the figure. The system is excited at its base (the casing) by an imposed displacement $y$, as illustrated in Figure 1(b), so that the suspended mass can oscillate inside its casing. A viscous damper $c$ is introduced as a generic dissipative term, which is a practical assumption validated by some recent experimental works on similar suspension configurations [17-19]. The relative displacement between the mass and the casing is indicated by $z$, so that when $z=0$ the mass is located at the centre of the casing. The system is subject to the acceleration of gravity $g$, pointing downwards, as indicated in the figure. Due to symmetry, the mass oscillation is assumed to be constrained to the vertical direction, and when the mass moves, the springs rotate changing their length. It is considered that $z_{0}$ is the initial displacement achieved upon assembly, as highlighted in Figure 1(a).

2.1. General Characteristic. The relationships between the mass displacement $z$ and the applied static elastic restoring force $f_{s}$ is given by

$$
f_{s}(z)=-2 k_{1}(a-z)\left(1-\sigma_{1} \frac{\sqrt{b^{2}+\left(a-z_{0}\right)^{2}}}{\sqrt{b^{2}+(a-z)^{2}}}\right)+\cdots+2 k_{2}(a+z)\left(1-\sigma_{2} \frac{\sqrt{b^{2}+\left(a+z_{0}\right)^{2}}}{\sqrt{b^{2}+(a+z)^{2}}}\right)+m g
$$

where $\sigma_{1}\left(\sigma_{2}\right)$ is a natural length factor, which accounts for the top (bottom) springs to be assembled in tension or compression respect to their corresponding natural length. This latter is obtained as the geometric distance between each spring attachments at $z=z_{0}$. The spring natural length factor is less (greater) than 1 when the springs are assembled in tension (compression), and it is equal to 1 when the springs are not deformed upon assembly.

Equation (1) may be conveniently rewritten in nondimensional form as

$$
\hat{f}_{s}(\widehat{z})=-2 \widehat{k}(1-\widehat{z})\left(1-\sigma_{1} \sqrt{\frac{\widehat{b}^{2}+\left(1-\widehat{z}_{0}\right)^{2}}{\widehat{b}^{2}+(1-\widehat{z})^{2}}}\right)+\cdots+2(1+\widehat{z})\left(1-\sigma_{2} \sqrt{\frac{\widehat{b}^{2}+\left(1+\widehat{z}_{0}\right)^{2}}{\hat{b}^{2}+(1+\widehat{z})^{2}}}\right)+\widehat{p},
$$

where $\hat{f}_{s}(\widehat{z})=\left(f_{s}(z) / a k_{2}\right), \quad \widehat{k}=\left(k_{1} / k_{2}\right), \quad \widehat{b}=(b / a)$, $\widehat{z}_{0}=\left(z_{0} / a\right), \widehat{z}=(z / a)$, and $\widehat{p}=\left(m g / a k_{2}\right)$.

Equation (2) is a highly nonlinear function of the nondimensional displacement $\widehat{z}$, and its parameters greatly affect the shape of the force-displacement characteristic. As a qualitative indication of the different shapes that the forcedeflection curve may assume, equation (2) is plotted for different values of the system parameters in Figures 2(a)$2(\mathrm{f})$, for displacements values in the range $-1.2<\bar{z}<1$. . In all subfigures, the solid lines correspond to the case with $\widehat{k}=\sigma_{1}=\sigma_{2}=\widehat{b}=\widehat{z}_{0}=\widehat{p}=1$, i.e., the case where the top springs are horizontal in absence of gravity, all springs have equal stiffness, and the casing is as wide as high. Dashed and dotted lines denote variations on one of the parameters, as indicated in each subfigure.

From Figure 2(a), it can be noted that as the stiffness ratio $\widehat{k}$ increases, i.e., the springs at the top become stiffer compared with those at the bottom, this generates a more symmetric behaviour for displacement values around zero. A similar qualitative trend is observed in Figure 2(b) (Figure 2(c)) when the natural length factor $\sigma_{1}\left(\sigma_{2}\right)$ increases (decreases), as these factors have a strengthening effect respect to a change in the corresponding stiffness. As shown in Figure $2(\mathrm{~d})$, the effect of decreasing the aspect ratio $\widehat{b}$ determines an increase of the nonlinear effect as the springs tend to rotate towards the vertical configuration. The effect 


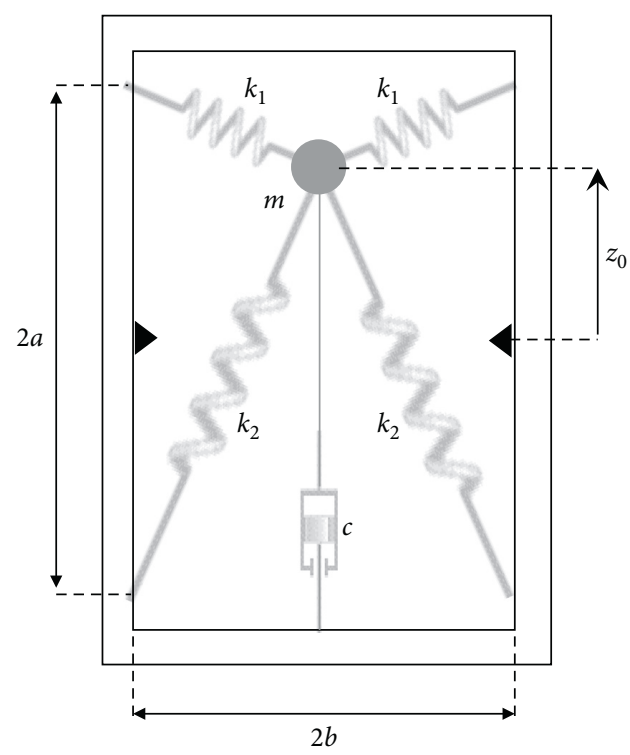

(a)

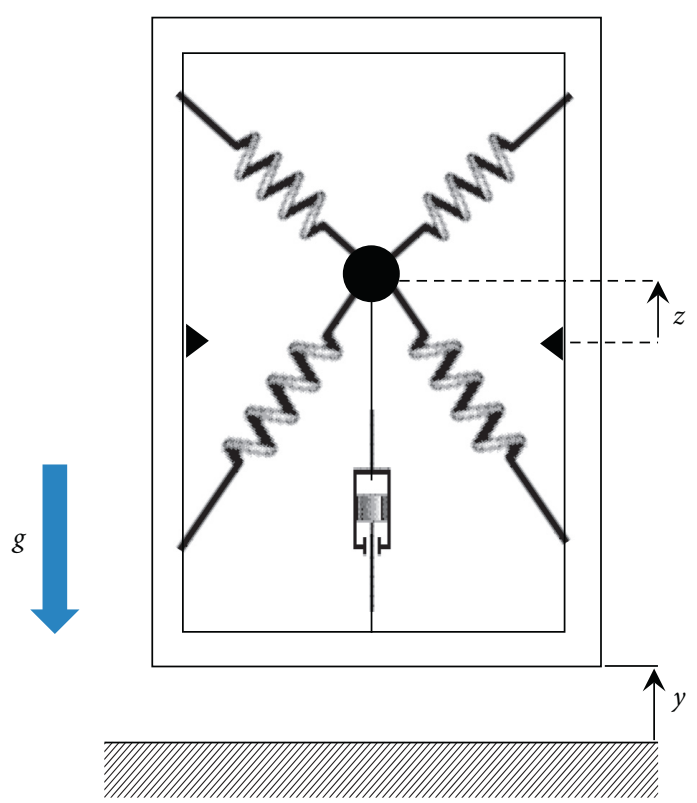

(b)

FIGURE 1: Schematic of the $X$-shaped suspension: (a) configuration upon assembly with no gravity effect; (b) generic deformed configuration subject to gravity.

of the initial deflection $\widehat{z}_{0}$ is to fundamentally shift the forcedeflection curve mainly along the horizontal axis in a nonlinear manner, as shown in Figure 2(e), while the effect of the nondimensional weight $\widehat{p}$ is to shift the force-deflection curve vertically, as illustrated in Figure 2(f).

Although the analysis performed above, based on a simple observation of Figure 2, is not exhaustive and a more advanced sensitivity study would be required for a deeper investigation, Figure 2 basically illustrates the qualitative change in the shape of the force-deflection curve, as each parameter is varied respect to a given reference configuration.
2.2. Symmetric Characteristic. In fact, the actual objective of this paper is to consider a particular shape of such static force-deflection curve, which motivates the qualitative analysis performed in the previous section. In particular, the interest is towards the case where the system has a symmetric behaviour around the static equilibrium configuration, and the static equilibrium configuration is achieved at $z=0$. Such a behaviour is achieved when the force-deflection curve is an odd function of the nondimensional displacement. To impose this condition, equation (2) is expanded in Taylor series around $z=0$, and the zero- and second-order coefficients are set to zero yielding

$$
\begin{aligned}
& -2 \hat{k}\left(1-\sigma_{1} \sqrt{\left.\frac{\widehat{b}^{2}+\left(1-\widehat{z}_{0}\right)^{2}}{\hat{b}^{2}+1}\right)+2\left(1-\sigma_{2} \sqrt{\frac{\widehat{b}^{2}+\left(1+\widehat{z}_{0}\right)^{2}}{\widehat{b}^{2}+1}}\right)+\widehat{p}=0,}\right. \\
& \qquad \frac{3 \hat{b}^{2}\left(\widehat{k} \sigma_{1} \sqrt{\hat{b}^{2}+\left(1-\widehat{z}_{0}\right)^{2}}-\sigma_{2} \sqrt{\hat{b}^{2}+\left(1+\widehat{z}_{0}\right)^{2}}\right)}{\left(1+\widehat{b}^{2}\right)^{5 / 2}}=0 . \\
& \qquad \hat{k}=\frac{2+\widehat{p}}{2}, \\
& \text { 4) are solved in terms of the } \\
& \text { natural length factor } \sigma_{2} \text { yielding } \\
& \text { relations among the system } \\
& \sigma_{2}=\sigma_{1} \frac{\sqrt{\hat{b}^{2}+\left(1-\widehat{z}_{0}\right)^{2}}(2+\widehat{p})}{2 \sqrt{\hat{b}^{2}+\left(1+\widehat{z}_{0}\right)^{2}}},
\end{aligned}
$$

Equations (3) and (4) are solved in terms of the stiffness ratio $\widehat{k}$ and the natural length factor $\sigma_{2}$ yielding the following simple relations among the system parameters: 

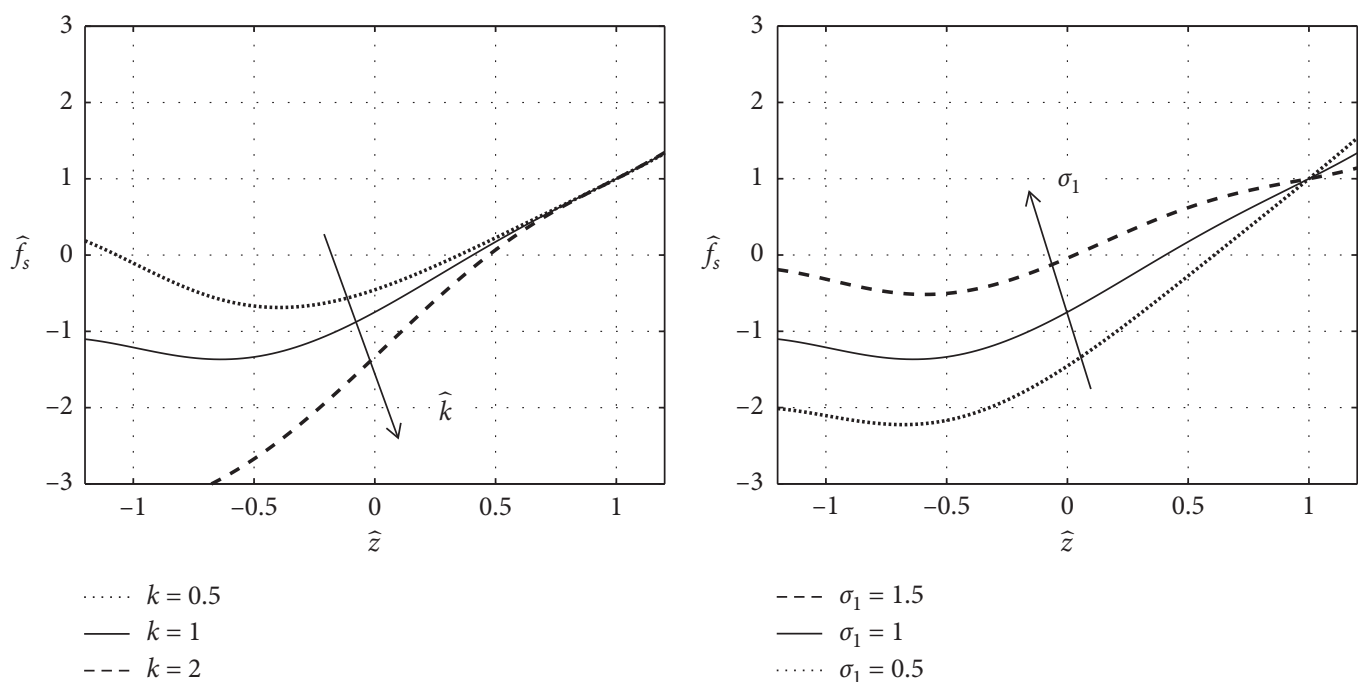

(a)

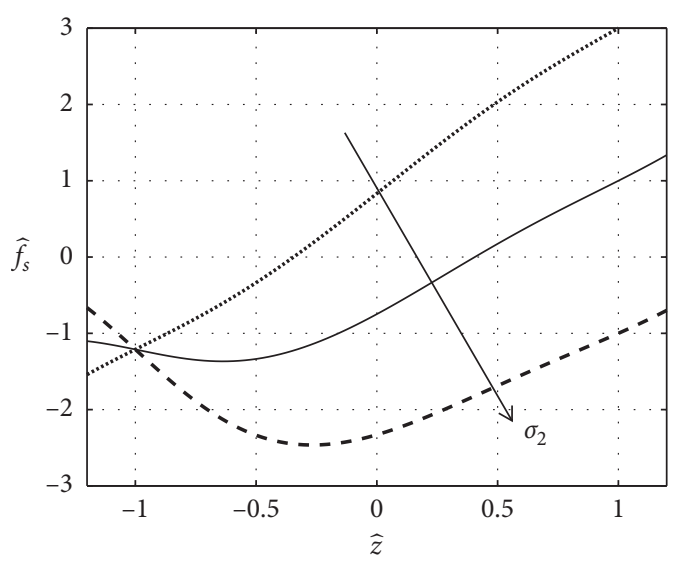

(b)

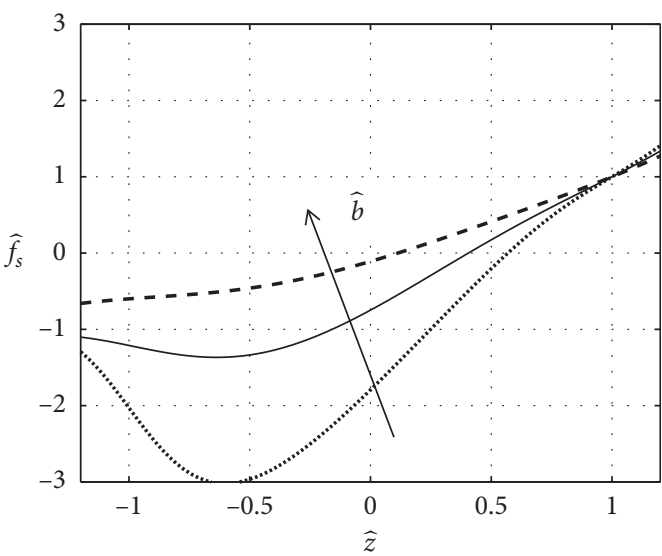

$\begin{aligned} \cdots \cdots & \sigma_{2}=0.5 \\ - & \sigma_{2}=1 \\ --- & \sigma_{2}=1.5\end{aligned}$

$---b=1.5$

$-b=1$

$b=0.5$

(c)

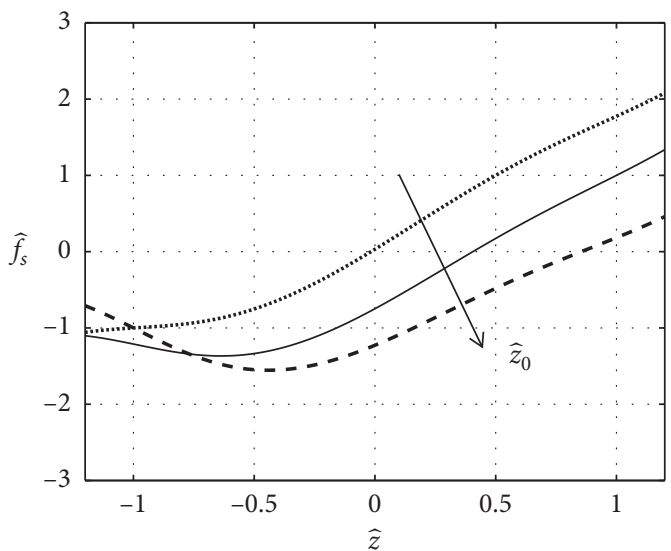

(d)

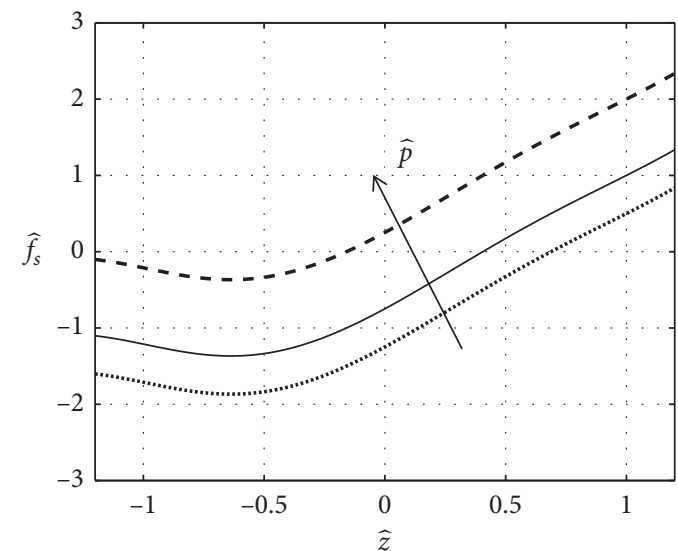

$$
\begin{aligned}
\cdots \cdots & z_{0}=0.5 \\
- & z_{0}=1 \\
--- & z_{0}=1.5
\end{aligned}
$$$$
\begin{aligned}
& --p=2 \\
& -p=1 \\
& \cdots \cdots p=0.5
\end{aligned}
$$

(e)

(f)

FIgURE 2: Nondimensional spring restoring force as a function of the nondimensional displacement for different values of the system parameters $(a-f)$, as indicated in the figure legends. The solid line is a common reference to all subfigures and corresponds to the case where $\widehat{k}=\sigma_{1}=\sigma_{2}=\widehat{b}=\widehat{z}_{0}=\widehat{p}=1$. 
which can be substituted back into equation (2) resulting in the following expression of the static force:

$$
\% \widehat{f}_{s}(\widehat{z})=-(2+\widehat{p})(1-\widehat{z})\left(1-\sigma_{1} \sqrt{\frac{\widehat{b}^{2}+\left(1-\widehat{z}_{0}\right)^{2}}{\widehat{b}^{2}+(1-\widehat{z})^{2}}}\right)+\cdots+2(1+\widehat{z})\left(1-\sigma_{1} \frac{(2+\widehat{p})}{2} \sqrt{\frac{\widehat{b}^{2}+\left(1-\widehat{z}_{0}\right)^{2}}{\widehat{b}^{2}+(1+\widehat{z})^{2}}}\right)+\widehat{p}
$$

Equation (7) only depends on the aspect ratio $\widehat{b}$, the nondimensional weight $\widehat{p}$, the natural length factor $\sigma_{1}$, and the initial deflection $\widehat{z}_{0}$, and it is plotted in Figures 3(a)-3(d) for different combinations of those four system parameters. In particular, the solid lines in all subfigures represent the case where $\sigma_{1}=\widehat{b}=\widehat{z}_{0}=1$.

From Figure 3, it is seen that the force-deflection curve is symmetric, with a general softening effect for large deflections. As the natural length factor $\sigma_{1}$ increases, as in Figure 3(a), the force-deflection curve exhibits a quasi-zero stiffness characteristic for large deflections, eventually leading to potential instability (negative stiffness). The effect of the aspect ratio $\widehat{b}$ is seen in Figure 3(b) and is similar to that in Figure 3(a). The effect of $\widehat{z}_{0}$ is illustrated in Figure 3(c), where it is seen that the curves are symmetric with respect to $\widehat{z}_{0}=1$. The effect of increasing $\widehat{p}$ is seen in Figure 3(d) and has an opposite effect respect to an increase in the parameters $\sigma_{1}$ and $\widehat{b}$ in Figures 3(a) and 3(b), respectively.

2.3. Symmetric Characteristic with QZS Behaviour. With the objective to further reduce the system complexity and the number of independent parameters, it is decided to investigate the specific conditions which yields to a QZS behaviour at large deflections (around $\widehat{z} \approx \pm 1$ ), followed by a increase in stiffness. Such a situation would guarantee that the mass oscillation will have a global positive stiffness characteristic.

From a mathematical point of view, the condition above is assured when both the first and second derivative of equation (7) are zero, which are the conditions for the appearance of an inflection point with horizontal tangent. Closed-form relationships among the system parameters to attain such a condition were not possible to be achieved; hence, numerical solutions were sought for some combinations of the system parameters. From a first insight, it can be demonstrated that the second derivative of equation (7), respect to the nondimensional displacement, is only dependent on the aspect ratio $\widehat{b}$, so that a curve is plotted in Figure 4 , to show the relation between $\widehat{b}$ and $\widehat{z}$ for $\left(\partial^{2} / \partial \widehat{z}^{2}\right) \widehat{f}_{s}=0$.

This curve is symmetric with respect to the vertical axis, so that only the values for $\bar{z}>0$ are plotted. It may be seen that for $\widehat{b}<1$, the inflection point in the force-deflection curve is around $\widehat{z}=1$ and dramatically decreases for $\widehat{b}>1$. The system of equations $\left(\partial^{2} / \partial \widehat{z}^{2}\right) \widehat{f}_{s}=(\partial / \partial \widehat{z}) \widehat{f}_{s}=0$ is then numerically solved in terms of $\widehat{z}$ and $\widehat{b}$, for fixed values of $\sigma_{1}$ and different combinations of $\widehat{z}_{0}$ and $\hat{p}$, varying in the range $0.1<\widehat{z}_{0}<0.9$ and $0<\widehat{p}<2$. Results are presented in terms of contour plots in Figure 5, which may be used for design purposes.

Figure 5 consists of a table of six panels organized in three rows and two columns, and it should be read as follows: the plots in each of the three rows refer to a different value of $\sigma_{1}$, while the first and second column show the solution for the inflection point in the force-displacement curve in terms of $\widehat{z}$ and $\widehat{b}$, respectively, as a function of $\widehat{z}_{0}$ and $\hat{p}$. It is noted that the inflection point is achieved for displacement values very close to 1 in all cases, while the aspect ratio generally decreases for increasing values of $\widehat{z}_{0}$.

Figure 6 shows the corresponding values of the natural length factor $\sigma_{2}$ and the force $\widehat{f}_{s}$ at the inflection point, obtained from Equations (6) and (7), respectively. For $\sigma_{1}<1$, it can be seen how $\sigma_{2}$ increases for increasing values of $\hat{p}$ and decreasing values of $\widehat{z}_{0}$, while $\widehat{f}_{s}$ at the inflection point fundamentally increases for increasing values of $\widehat{z}_{0}$. The change in $\widehat{f}_{s}$ for changing values of $\widehat{z}_{0}$ is opposite for the case when $\sigma_{1}>1$ than for the case when $\sigma_{1}<1$.

As application examples, two different spring configurations are designed using the aid of Figures 5 and 6. In a first case, it is assumed that $\sigma_{1}=1, \widehat{z}_{0}=0.4$, and $\widehat{p}=1.5$, which corresponds to the circle () in Figures 5 and 6. In particular, from Figure 5(c) it is noted that the inflection point occurs at about 1; from Figure 5(d), it can be noted that the aspect ratio is about $\widehat{b}=0.5$, while from Figure 6(c) and Figure 6(d), respectively, it is noted that to achieve such configuration it should be set $\sigma_{2}=0.9$ approximately, and the expected value of the force $\widehat{f}_{s}$ at the inflection point is about 2.8 . The forcedisplacement curve corresponding to this case is plotted from equation (7) in Figure 7(a) as a thick solid curve (other curves will be described further below).

In a second case, it is assumed that it is of interest to suspend a mass with nondimensional weight $\widehat{p}=1$ and to set the nondimensional force $\widehat{f}_{s}$ at the inflection point at about 2 . We have different design configurations to achieve such requirements. However, if we want the springs at the bottom to be as unloaded as possible upon initial assembly, we can note that the solution indicated by the square () in Figures 5 and 6 would fit the case and corresponds to about $\sigma_{1}=1.2, \widehat{z}_{0}=0.4$, $\widehat{b}=0.65$, and $\sigma_{2}=1$. The corresponding force-deflection curve is plotted in Figure 7(b) as a thick solid line (other curves will be described further below). Animation S1in the Supplementary Material illustrates the corresponding physical assembly subject to a quasi-static test.

The force-deflection curve corresponding to the diamond () and star () in Figures 5 and 6 is shown in Figures $7(\mathrm{c})$ and $7(\mathrm{~d})$, respectively, as a thick solid curve (other curves will be described further below). 


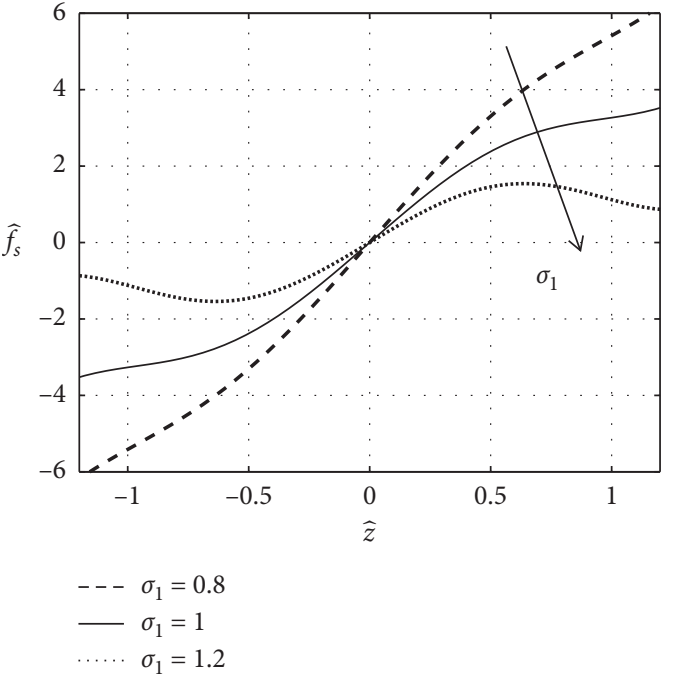

(a)

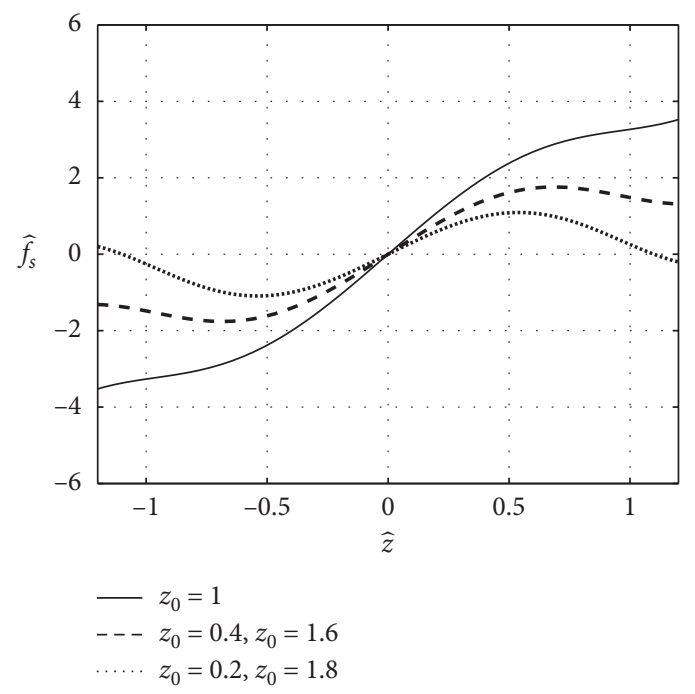

(c)

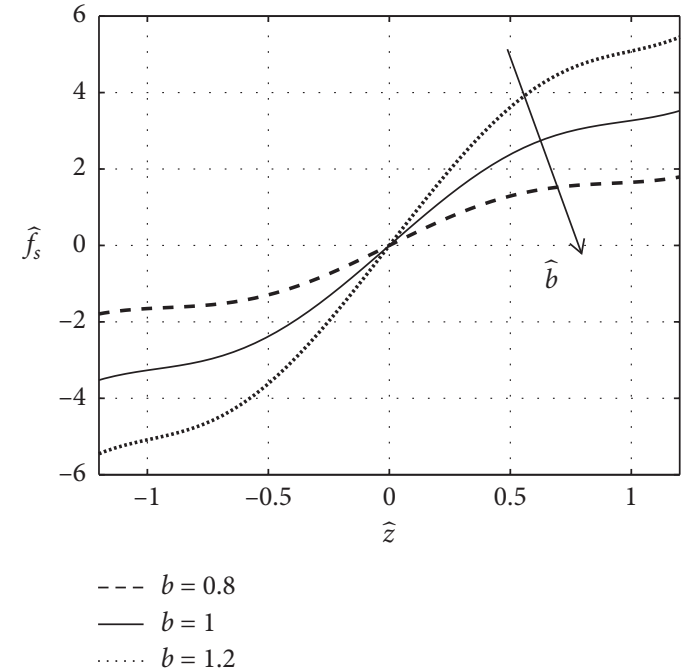

(b)

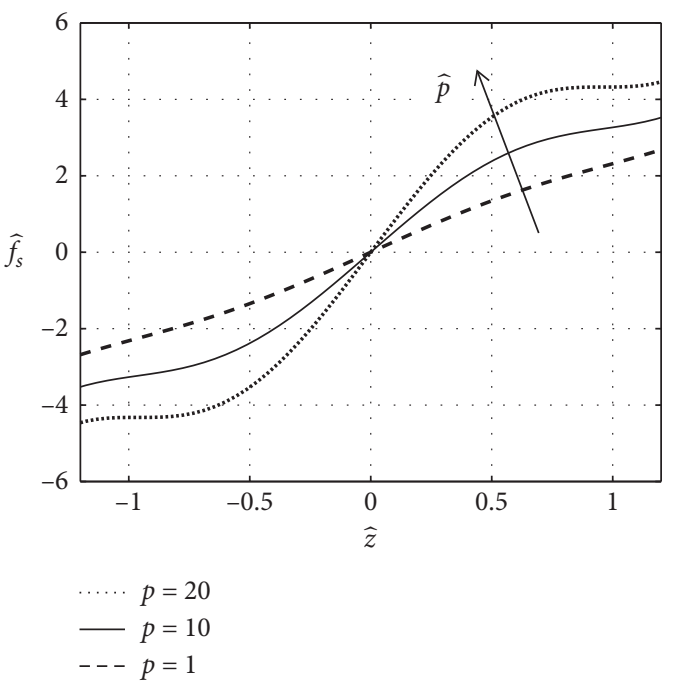

(d)

Figure 3: Symmetric force-deflection curve for different values of the system parameters (a-d), as indicated in the figure legends. The solid line is the common reference to all subfigures and corresponds to the case where $\sigma_{1}=\widehat{b}=\widehat{z}_{0}=1$ and $\widehat{p}=10$.

\section{Dynamic Analysis}

With the aim of incorporating the static force-deflection curve with QZS characteristic at large displacements into the dynamic equation of motion of the system and performing an analytical insight, equation (7) is approximated by a polynomial expression. Unfortunately, the classical Taylor series expansion around the static equilibrium configuration would have the limitation that a high order solution would be needed to fit the QZS behaviour at large deflections [16], i.e., far away from the expansion point of the series. To illustrate this, the results of a 7th order Taylor series expansion are illustrated in Figures $7(\mathrm{a})-7(\mathrm{~d})$ as thin dotted lines and compared with the corresponding curve from equation (7), as thick solid lines. It can be observed that a negative stiffness is predicted for values of displacements greater (smaller) than $1(-1)$.

To overcome such limitation, the force-deflection curve in equation (7) is approximated by a polynomial expression with the following conditions: (i) the stiffness for $\widehat{z}=0$ is set equal to that from Taylor series; (ii) the stiffness at $\widehat{z}=1$ (the approximate location of the inflection point) is set to zero; (iii) the derivative of the stiffness at $\widehat{z}=1$ is set to zero; and (iv) the force at $\widehat{z}=1$ is set equal to that from equation (7). To satisfy these conditions, the following 7 th order polynomial expression is obtained:

$$
\widehat{f}_{s, \text { approx }}(\widehat{z})=\widehat{k}_{1} \widehat{z}+\widehat{k}_{3} \widehat{z}^{3}+\widehat{k}_{5} \widehat{z}^{5}+\widehat{k}_{7} \widehat{z}^{7},
$$

where 


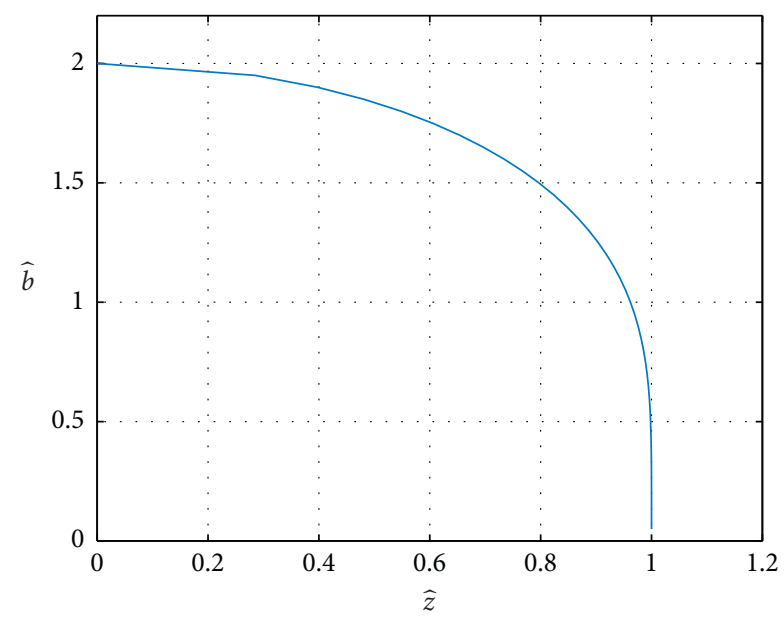

FIgURE 4: Relation between the aspect ratio $\widehat{b}$ and the displacement $\widehat{z}$ at the inflection point in case of a force-deflection curve exhibiting QZS behaviour.

$$
\begin{aligned}
& \widehat{k}_{1}=(4+\widehat{p})-2 \widehat{b}^{2} \sigma_{1}(2+\widehat{p}) \sqrt{\widehat{b}^{2}+\left(1-\widehat{z}_{0}\right)^{2}}\left(1+\widehat{b}^{2}\right)^{-(3 / 2)}, \\
& \widehat{k}_{3}=\frac{35}{8} \widehat{f}_{p}-3 \widehat{k}_{1}, \\
& \widehat{k}_{5}=-\frac{21}{4} \widehat{f}_{p}+3 \widehat{k}_{1}, \\
& \widehat{k}_{7}=\frac{15}{8} \widehat{f}_{p}-\widehat{k}_{1}, \\
& \widehat{f}_{p}=\widehat{f}_{s}(\widehat{z}=1)=4\left(1-\sigma_{1} \frac{(2+\widehat{p})}{2} \sqrt{\frac{\widehat{b}^{2}+\left(1-\widehat{z}_{0}\right)^{2}}{\hat{b}^{2}+4}}\right)+\widehat{p} .
\end{aligned}
$$

Equation (8) is plotted in Figures $7(a)-7(d)$ as a dashed line, and it can be noted that such approximate expression fits the exact force-deflection curve (solid line) better than that from the Taylor series approximation (dotted line). Furthermore, the presence of a negative stiffness behaviour is avoided for nondimensional displacement values around \pm 1 . The lower panels in each subfigure show the residual errors of the Taylor series and polynomial fitting approximation.

However, from Figures $7(\mathrm{a})-7(\mathrm{~d})$, it can be seen that when $|\widehat{z}|$ becomes greater than 1 , the polynomial fitting error rapidly increases. In the following analysis, it is thus assumed that the approximate working condition of the suspension is such that $|\widehat{z}| \leq 1.3$, where it is seen that the residual error of the polynomial fitting approximation is below $10 \%$.

3.1. Amplitude-Frequency Equation. The equation of motion of the system depicted in Figure lis given by

$m \ddot{z}+c \dot{z}+f_{s}(z)=-m \ddot{y}$, where the static spring restoring force $f_{s}(z)$ is given by equation (1), and the overdots denote differentiation respect to time $t$. The oscillator is excited at the base by a harmonic displacement $y=Y \cos (\omega t+\varphi)$ where $Y$ is the displacement amplitude, $\omega$ is the angular frequency, and $\varphi$ is the phase.

By using the approximate expression for the spring restoring force given in equation (8), equation (10) may be conveniently written in nondimensional form as

$$
\widehat{z} \prime \prime+2 \zeta \widehat{z}^{\prime}+\widehat{z}+\gamma \widehat{z}^{3}+\delta \widehat{z}^{5}+\varepsilon \widehat{z}^{7}=\Omega^{2} \widehat{Y} \cos (\Omega \tau+\varphi),
$$

where $\tau=\omega_{0}$ tand $\Omega=\left(\omega / \omega_{0}\right)$ are the nondimensional time and frequency, respectively, $\omega_{0}=\sqrt{k_{2} \widehat{k}_{1} / m}, \zeta=\left(c / 2 m \omega_{0}\right)$ is the damping ratio, $\widehat{Y}=(Y / a)$ is the nondimensional displacement amplitude, primes denote differentiation respect to $\tau$, and

$$
\begin{aligned}
& \gamma=\frac{\widehat{k}_{3}}{\widehat{k}_{1}}=\frac{35}{8} \frac{\widehat{f}_{p}}{\widehat{k}_{1}}-3, \\
& \delta=\frac{\widehat{k}_{5}}{\widehat{k}_{1}}=-\frac{3}{5}-\frac{6}{5} \gamma, \\
& \varepsilon=\frac{\widehat{k}_{7}}{\widehat{k}_{1}}=\frac{2}{7}+\frac{3}{7} \gamma .
\end{aligned}
$$

The expressions given in equation (12) show that the fifth- and seventh-order coefficients are dependent on the cubic one. Both the statics and dynamics of the system exhibiting QZS behaviour for large deflections (at $|\widehat{z}|$ up to approximately 1.3) may be then described in terms of one key parameter only.

The values of $\gamma$ are calculated based on equation (12) and shown in the contour plots of Figure 8, in the same way as for Figures 5 and 6.

To solve equation (11) in closed form in terms of the amplitude-frequency equation, it is assumed that the system response is predominately harmonic at the excitation frequency, i.e., $\widehat{z}=\widehat{Z} \cos (\Omega \tau)$, and this is substituted back into equation (11), where a first-order harmonic balance approximation is applied to yield

$$
\Omega^{4}\left[\widehat{Z}^{2}-\widehat{Y}^{2}\right]+2 \Omega^{2} \widehat{Z}^{2}\left[2 \zeta^{2}-G\right]+\widehat{Z}^{2} G^{2}=0
$$

with $G=1+(3 / 4) \gamma \widehat{Z}^{2}+(5 / 8) \delta \widehat{Z}^{4}+(35 / 64) \varepsilon \widehat{Z}^{6}$.

3.2. Backbone Curve and Effect of Damping. The analytical amplitude-frequency equation reported in equation (13) can be used to investigate the backbone curve and the effect of damping on the system response.

First, the relation between the resonance peak and the corresponding frequency is obtained by setting the discriminant of equation (13) to zero yielding 


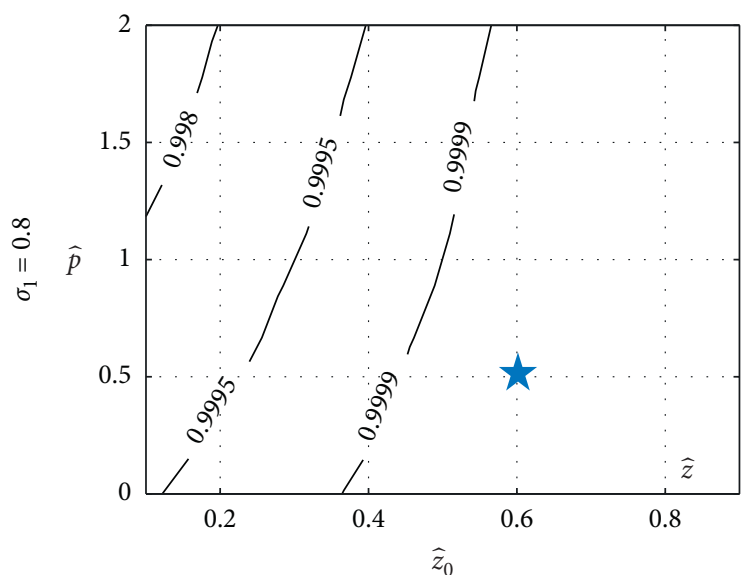

(a)

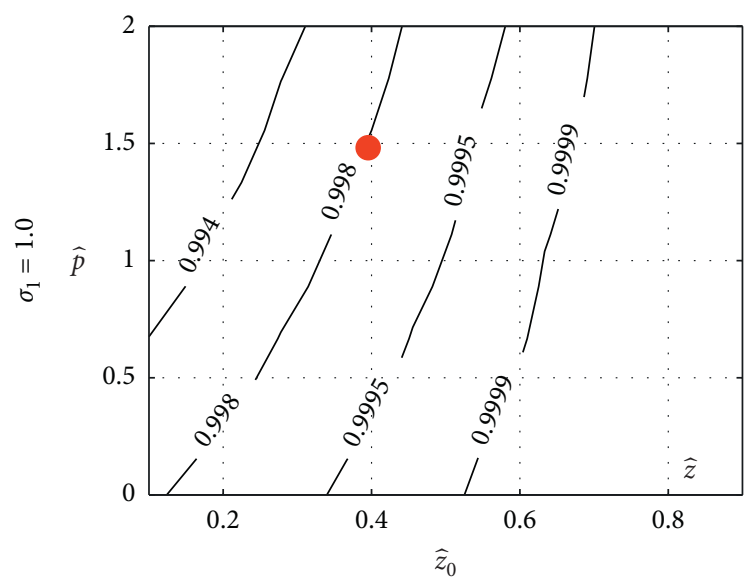

(c)

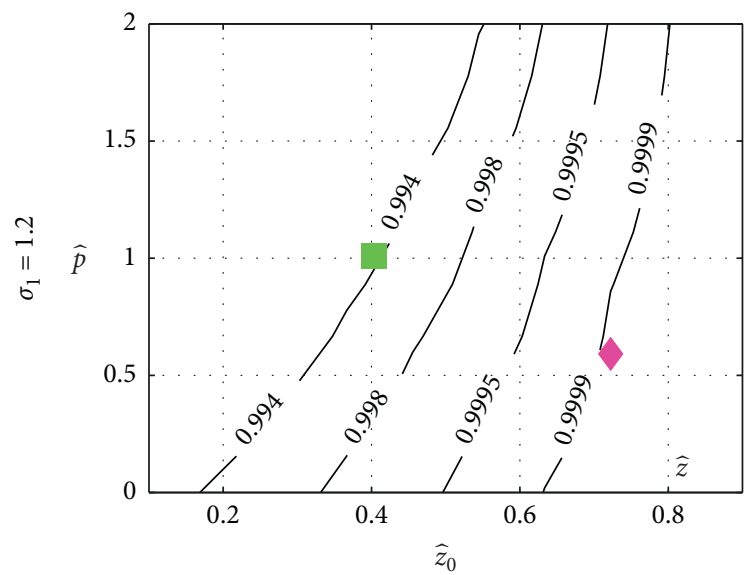

(e)

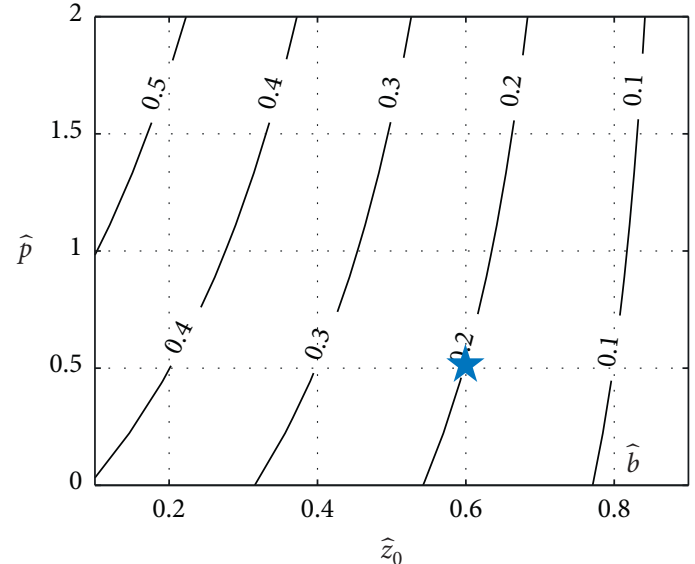

(b)

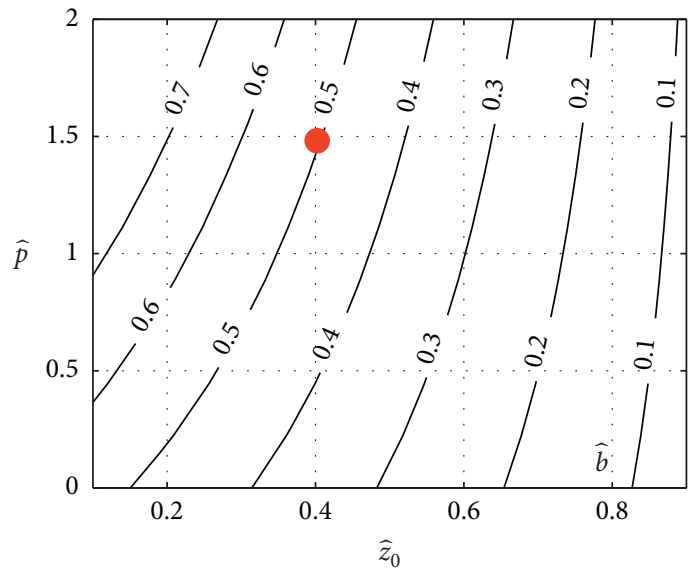

(d)

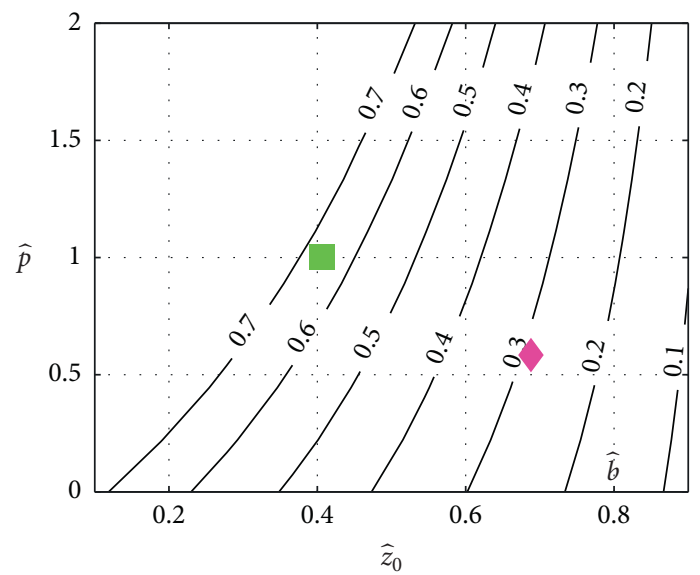

(f)

FIgURE 5: Contour plots for the design of an $X$-shaped suspension with QZS behaviour at large deflections: values of $\widehat{z}$ at the inflection point and $\widehat{b}$ for different combinations of $\sigma_{1}, \widehat{z}_{0}$, and $\widehat{p}$ (markers will be described later).

$$
4 \widehat{Z}^{4}\left[2 \zeta^{2}-G\right]^{2}-4 \widehat{Z}^{2} G^{2}\left[\widehat{Z}^{2}-\widehat{Y}^{2}\right]=0
$$

which can be solved in terms of the damping ratio squared to give

$$
\zeta_{\text {peak }}^{2}=\frac{G}{2}\left[1-\sqrt{1-\frac{\widehat{Y}^{2}}{\widehat{Z}^{2}}}\right]
$$




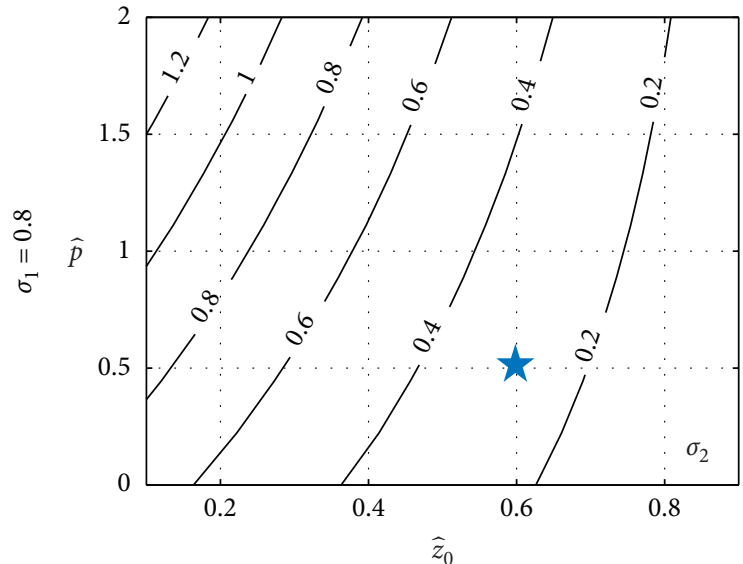

(a)

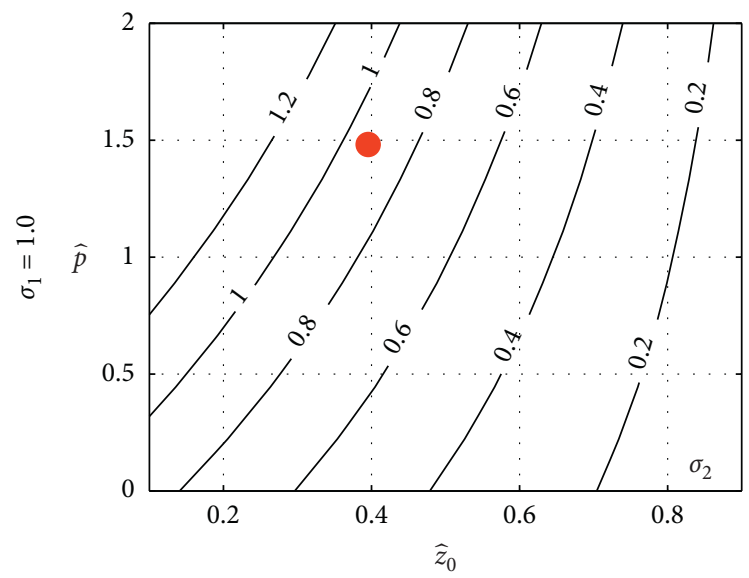

(c)

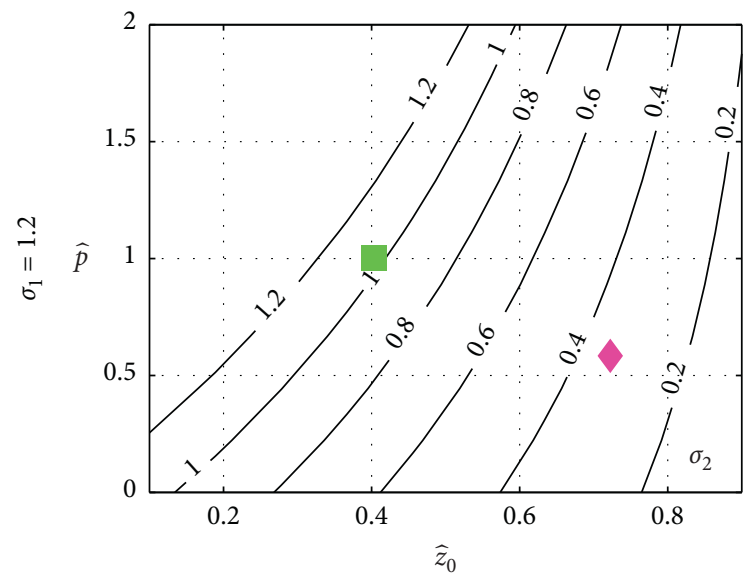

(e)

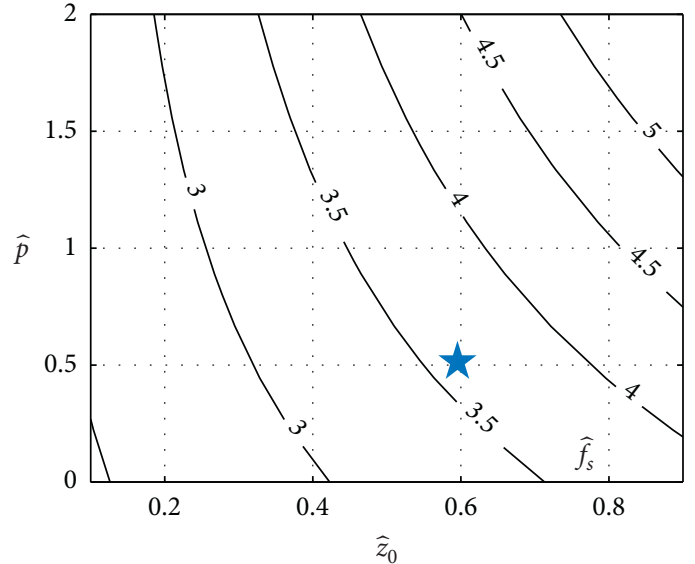

(b)

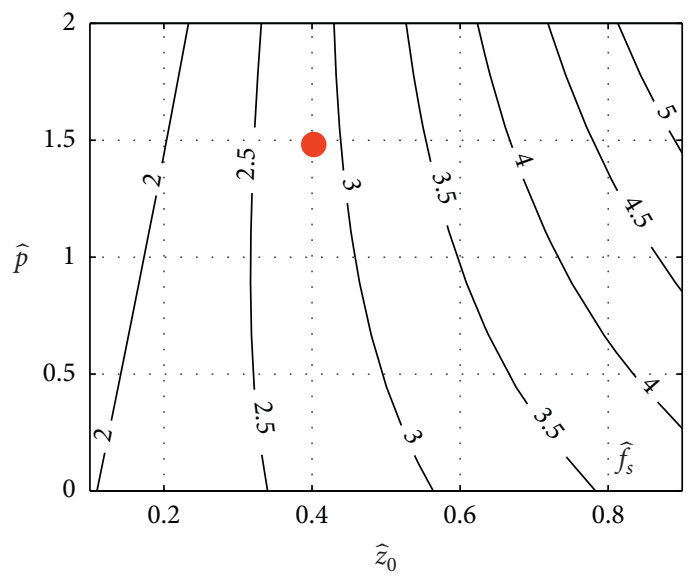

(d)

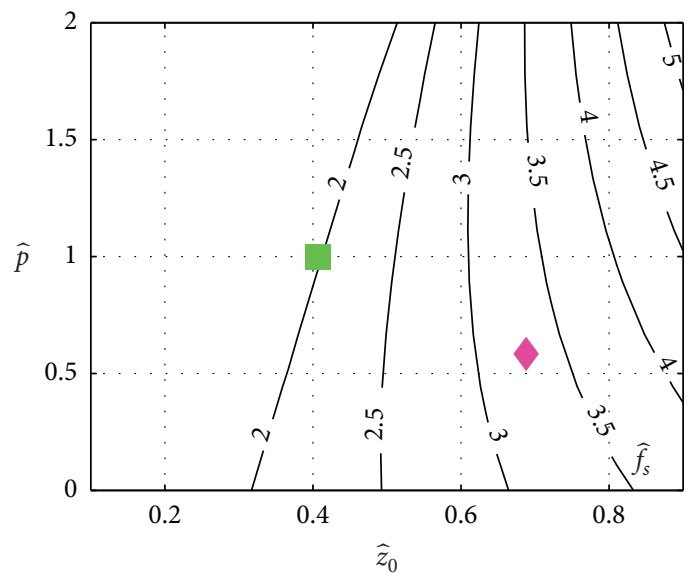

(f)

FIGURE 6: Contour plots for the design of an $X$-shaped suspension with QZS behaviour at large deflections: values of $\sigma_{2}$ and $\widehat{f}_{s}$ for different combinations of $\sigma_{1}, \widehat{z}_{0}$, and $\hat{p}$. (markers will be described later).

By combining equation (15) and equation (13), the expression for the backbone curve, i.e., the unforced and undamped response of the nonlinear oscillator is obtained as

$$
\Omega_{\text {backbone }}=\sqrt{G}=\frac{1}{8} \sqrt{64+48 Z^{2} \gamma-24 Z^{4}(1+2 \gamma)+5 Z^{6}(2+3 \gamma)} .
$$




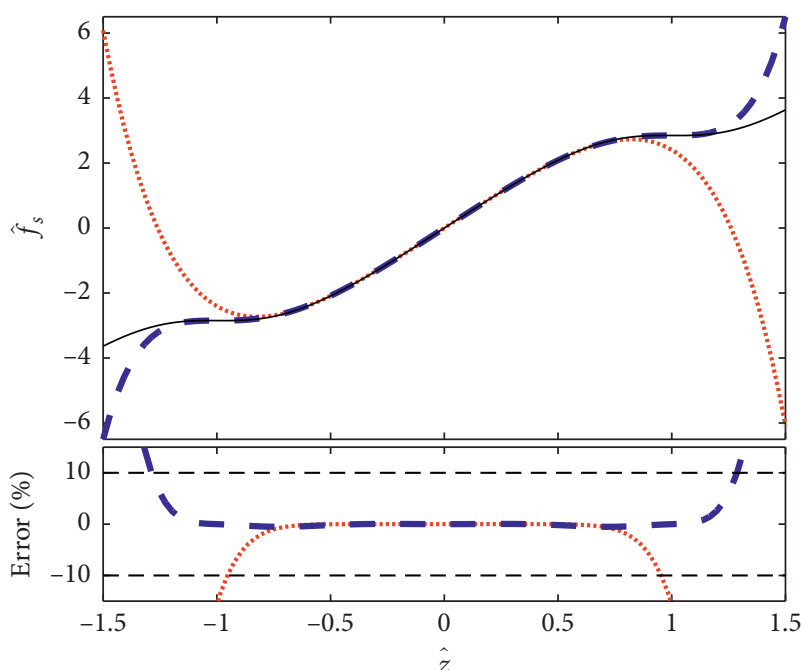

(a)

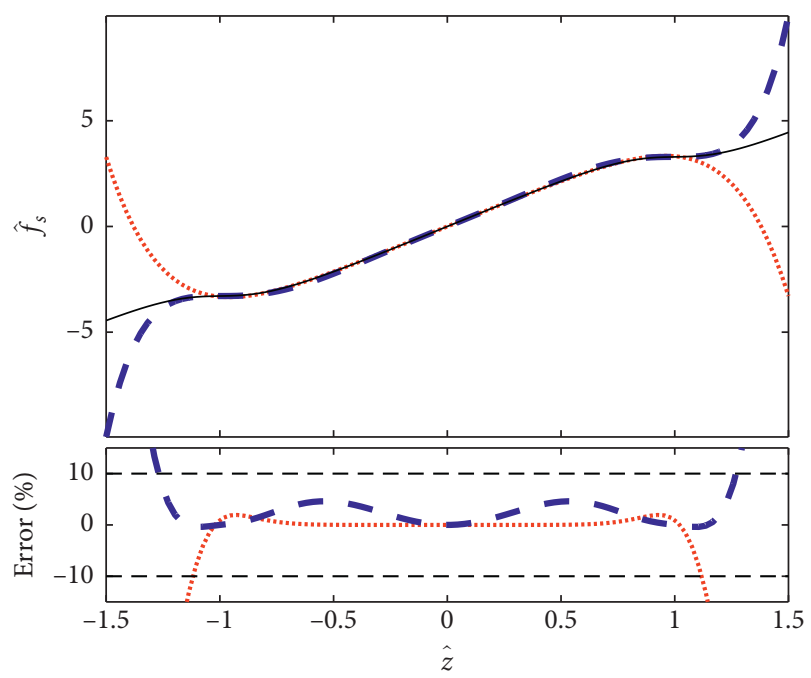

(c)

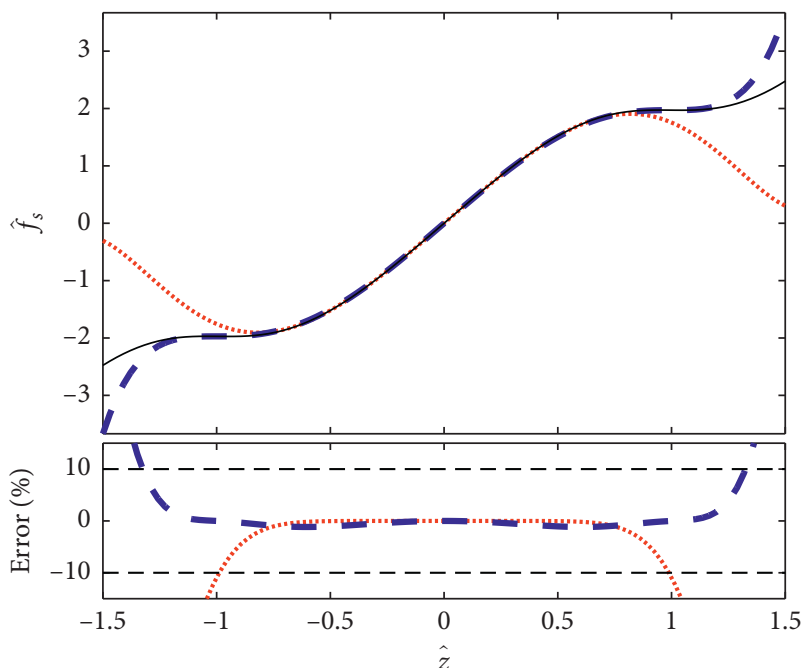

(b)

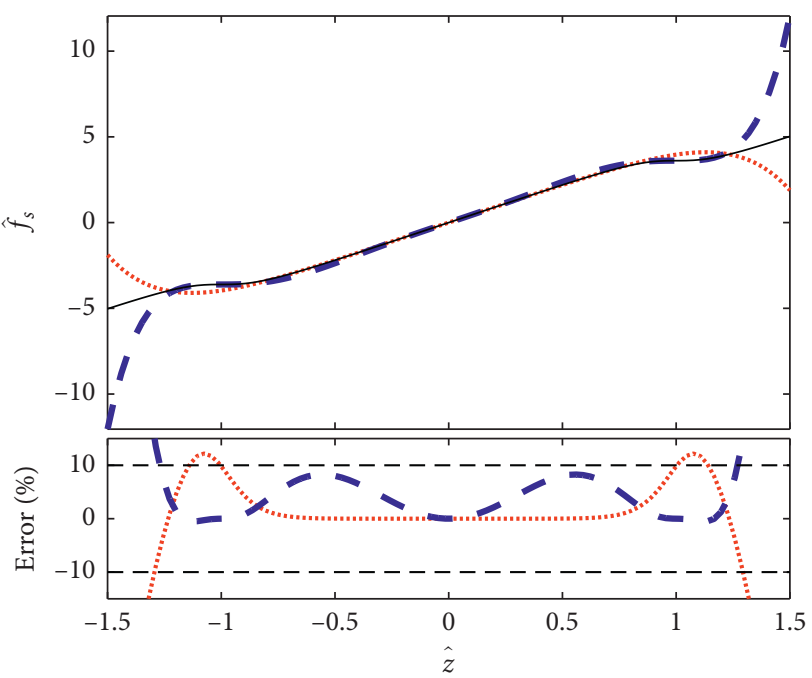

(d)

Figure 7: Force-displacement curve corresponding to different combinations of the system parameters as indicated by the (a) circle, (b) square, (c) diamond, and (d) star in Figures 5 and 6. Exact curves from equation (7) are represented as solid lines; Taylor series approximations are represented as dotted lines; and polynomial approximations using equation (8) are represented as dashed lines.

To qualitatively illustrate the shape of the backbone curve for the system considered in this work, equation (16) is plotted in Figure 9(a) as a thick line, for the different values of $\gamma$.

It can be noted that the frequency of the backbone curve may exhibit a relative minimum and maximum for certain values of $\gamma$. These are due to the transition among the softening and hardening stiffness characteristic in the spring force-deflection curve, and such effect is better investigated below. Of particular interest is the occurrence of a relative minimum in the backbone curve, as this leads to a minimum stable value for the resonance frequency.

An approximate expression for the displacement amplitude at the relative extremes of the resonance frequency in the backbone curve is obtained by differentiating equation (16) with respect to $\widehat{Z}$, equating to zero, and solving to give

$$
\widehat{Z}_{C, D}=2 \sqrt{\frac{2+4 \gamma \pm \eta}{5(2+3 \gamma)}}
$$

where $\eta=\sqrt{4+6 \gamma+\gamma^{2}}$ and $C(D)$ indicates the point where a relative minimum (maximum) occurs. The corresponding frequency is determined by substituting equation (17) back into equation (16) to yield

$$
\Omega_{C, D}=\frac{1}{5} \sqrt{\frac{84+264 \gamma+243 \gamma^{2}+52 \gamma^{3} \mp\left(8+12 \gamma+2 \gamma^{2}\right) \eta}{(2+3 \gamma)^{2}}} .
$$

The loci of points $C$ (relative minimum) and $D$ (relative maximum) are indicated in Figure 9(a) as a thin solid and dash-dotted line, respectively, while their frequency and 


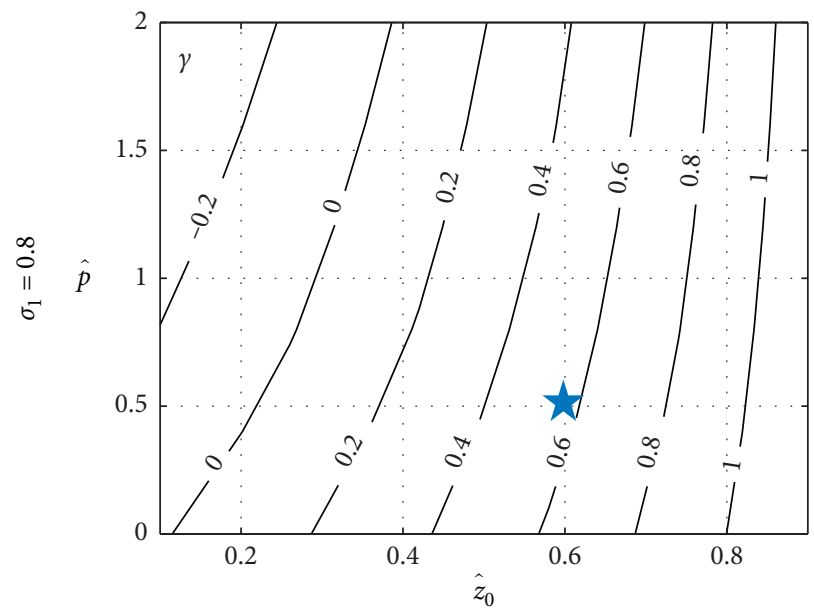

(a)

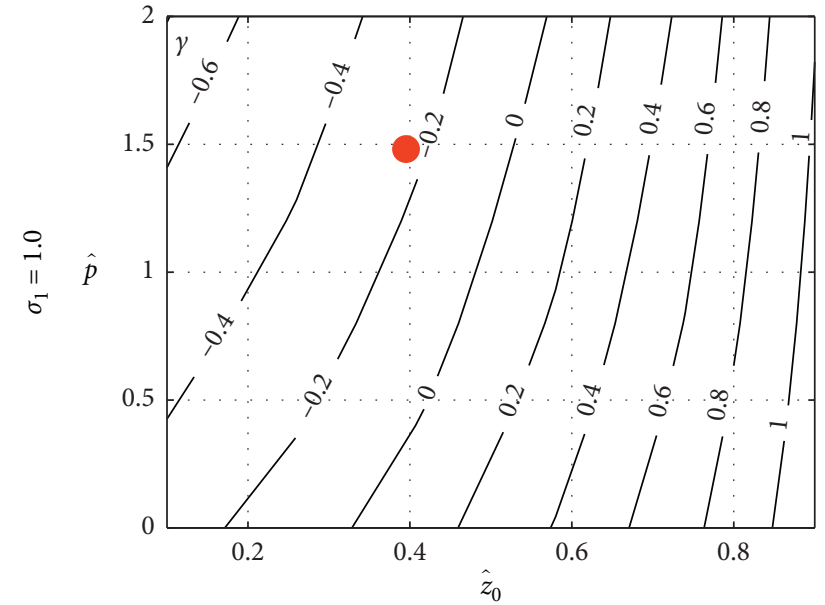

(b)

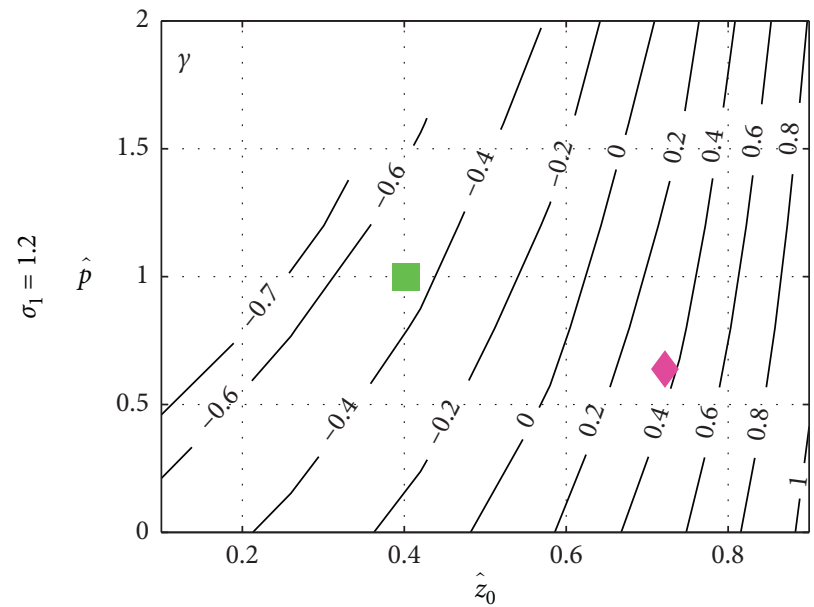

(c)

Figure 8: Contour plots for the design of an $X$-shaped suspension with QZS behaviour at large deflections: values of $\gamma$, for different combinations of $\sigma_{1}, \widehat{z}_{0}$, and $\widehat{p}$.

amplitude are plotted in Figures 9(b) and 9(c), respectively, as a function of $\gamma$.

Form Figure 9, it can be noted that for $\gamma>0$, the backbone curve presents a relative maximum frequency at a lower displacement amplitude and a relative minimum frequency at a larger displacement amplitude, so that a hardening behaviour manifests at larger amplitudes. In the range $-(2 / 3)<\gamma<0$, the backbone curve presents only a relative minimum frequency, with hardening behaviour at high frequency. In the range $-3+\sqrt{5}<\gamma \leq-(2 / 3)$, the backbone curve presents both a relative maximum and a relative minimum frequency, but now the relative maximum appears at a larger amplitude, so that a softening behaviour manifests at larger displacements, potentially leading to instability. For $\gamma=-3+\sqrt{5}$, the relative maximum and minimum frequencies coincide and an inflection point is formed. For $\gamma<-3+\sqrt{5}$, no relative maximum or minimum frequency occurs, and an overall softening behaviour manifests, potentially leading to instability for large motion.
The minimum frequency and corresponding displacement amplitude, which are indicated by the circle in Figures 9(b) and $9(\mathrm{c})$, are given, respectively, by $5^{-(1 / 4)} \approx 0.69$ and $\sqrt{2+(2 / \sqrt{5})} \approx 1.7$.

The values of $\Omega_{C}$ and $\widehat{Z}_{C}$ are calculated and shown in the contour plots of Figure 10, in the same way as for Figures 5 and 6. It can be noted that for a reasonable range of parameter values, the peak amplitude of the nondimensional displacement does not exceed the approximate value of 1.3, which validates the polynomial fitting approximation of the force-deflection curve presented above.

It is now possible to investigate the effect of damping on the system response. Of particular interest is the value of damping which leads to the minimum resonance frequency, corresponding to point $C$, discussed above. To this purpose, the ratio $\left(\zeta_{C} / \widehat{Y}\right)$ is calculated from equations (15) and (17), for different values of excitation amplitude, and is plotted in the contour plots of Figure 11. In Figure 11, dashed lines correspond to $\widehat{Y}=0.01$, solid lines correspond to $\widehat{Y}=0.3$, 


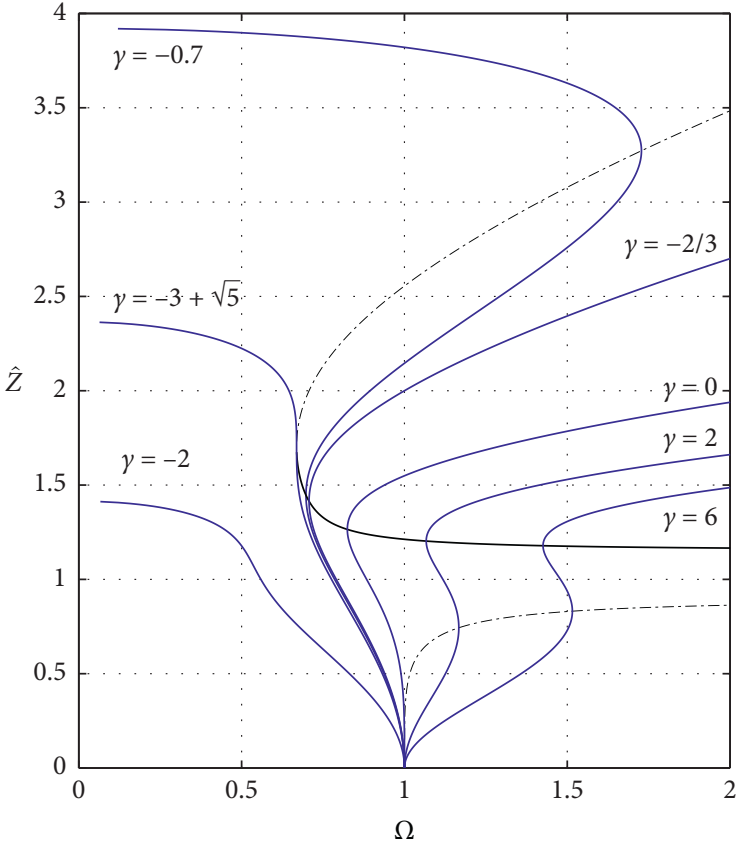

(a)

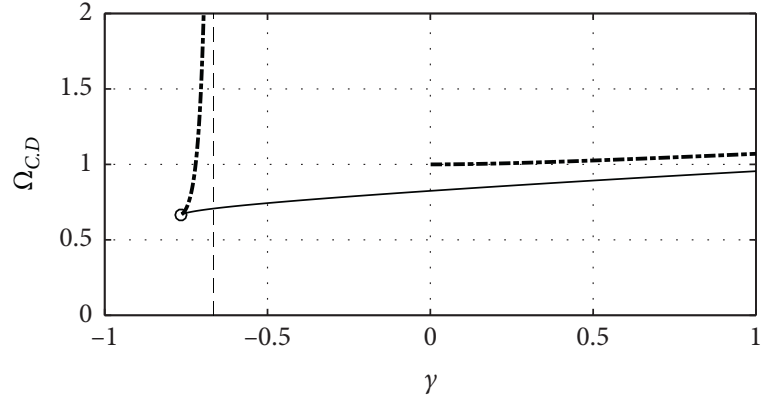

(b)

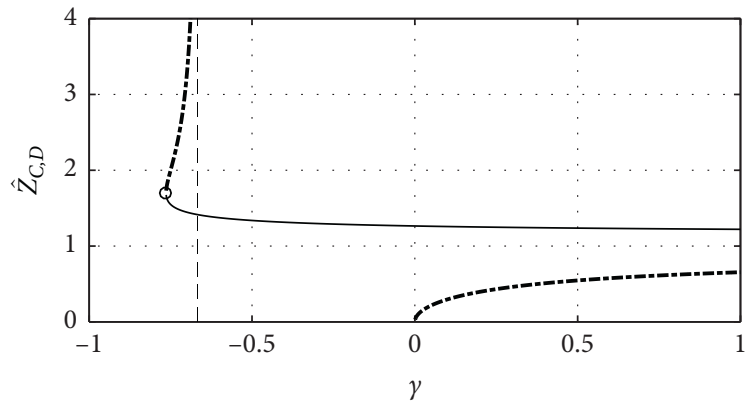

(c)

Figure 9: (a) Backbone curves (thick solid lines) for different values of $\gamma$, as labelled; locus of points C, i.e., relative minima, (thin solid line) and points D, i.e., relative maxima, (thin dash-dotted line). (b) Frequency corresponding to the relative minima (solid line) and maxima (dash-dotted line) as a function of $\gamma$. (c) Displacement amplitude corresponding to the relative minima (solid line) and maxima (dash-dotted line) as a function of $\gamma$. The vertical dashed line in (b) and (c) denotes the asymptotic trend for $\gamma=-(2 / 3)$, and the circle denotes the limit for $\gamma=-3+\sqrt{5}$.

and dash-dotted lines correspond to $\widehat{Y}=0.5$. As can be noted, despite the large variation in the amplitude of excitation, the ratio $\left(\zeta_{C} / \bar{Y}\right)$ does not change much and stays in the range between about 0.28 and 0.38 .

A closed-form expression can be obtained by expanding equation (15) in Taylor series for small excitation amplitudes and rearranging to give

$$
\frac{\zeta_{C}}{\widehat{Y}} \approx \frac{\sqrt{G}}{2 \widehat{Z}_{C}}=\frac{\Omega_{C}}{2 \widehat{Z}_{C}}
$$

which can be further expressed for small values of $\gamma$ as

$$
\frac{\zeta_{C}}{\widehat{Y}} \approx \frac{1}{4} \sqrt{\frac{17}{10}}+\frac{13}{64} \sqrt{\frac{5}{34}} \gamma
$$

This is a very simple closed-form expression and shows that the damping to achieve the minimum resonance frequency is proportional to the amplitude of excitation, through a coefficient which is a linear function of the parameter $\gamma$.

To validate the approximate expression in equation (20), this is plotted in Figure 12 as a dashed line and compared with the results from equation (15) for a relatively high and low value of excitation amplitude. It can be seen that despite the large variation of the excitation amplitude (10\% and 50\% of the displacement at the QZS point), the approximate expression captures the main trend for values of $\gamma$ greater that approximately -0.5 .

3.3. Frequency Response Curve. To better illustrate and validate the results presented above, the frequency response curves (FRCs) are plotted in Figures 13(a)-13(d) for two different values of the excitation amplitude and for the 


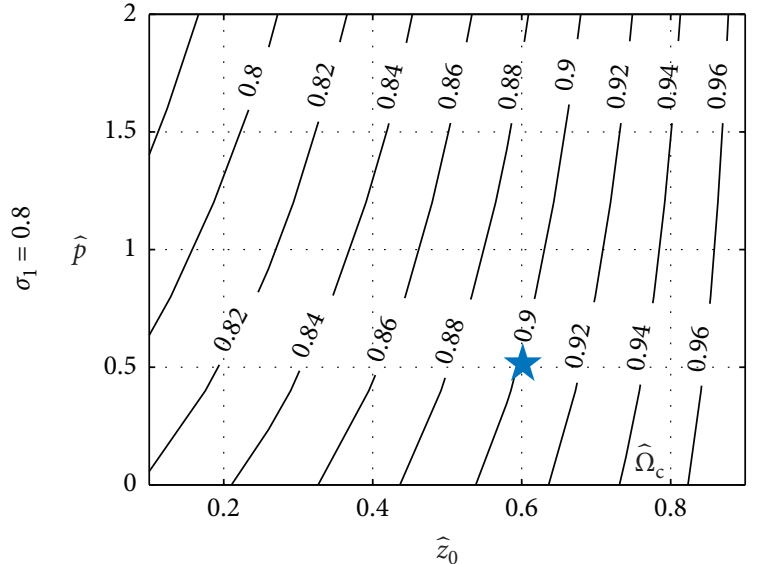

(a)

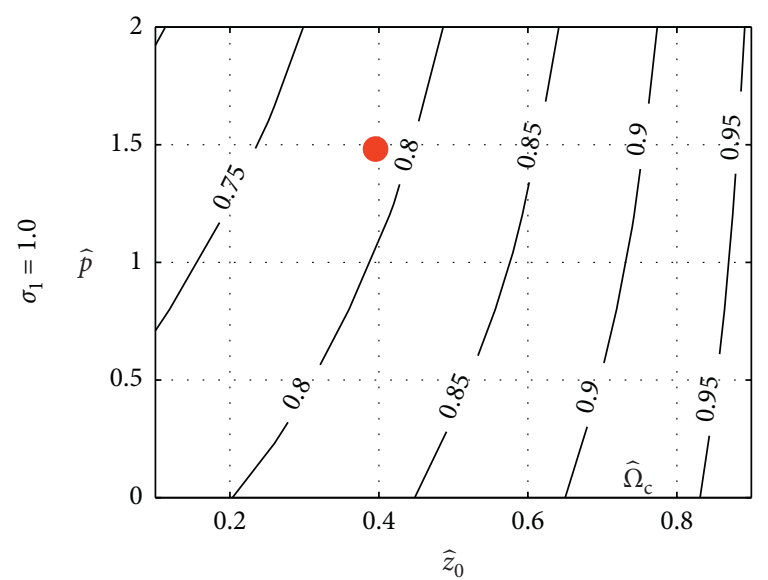

(c)

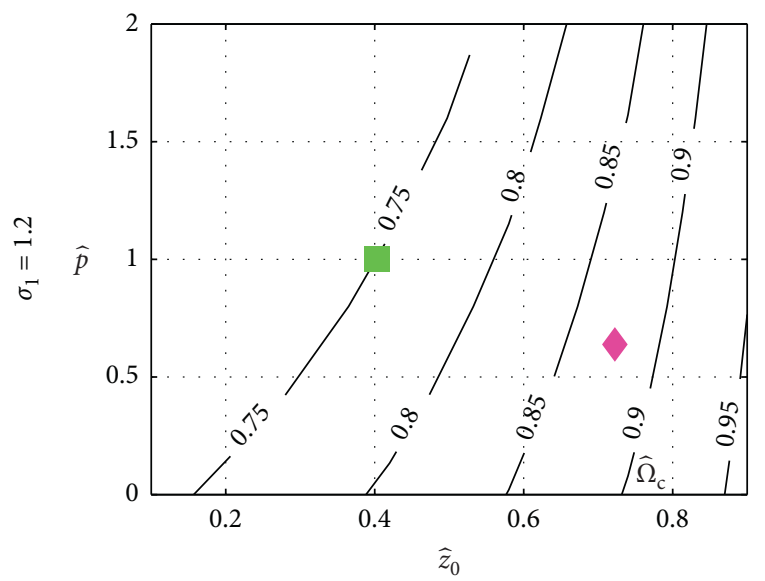

(e)

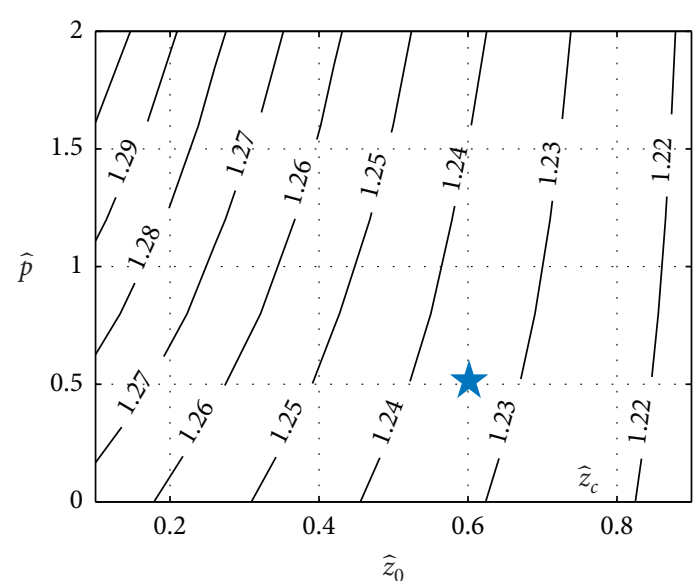

(b)

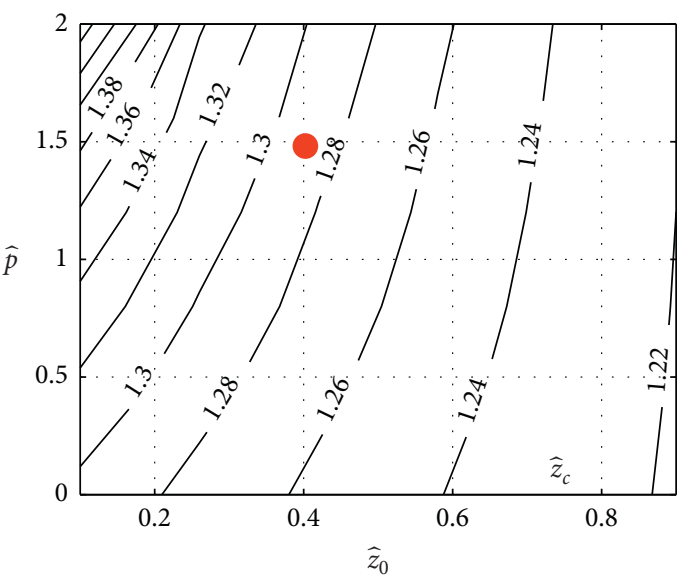

(d)

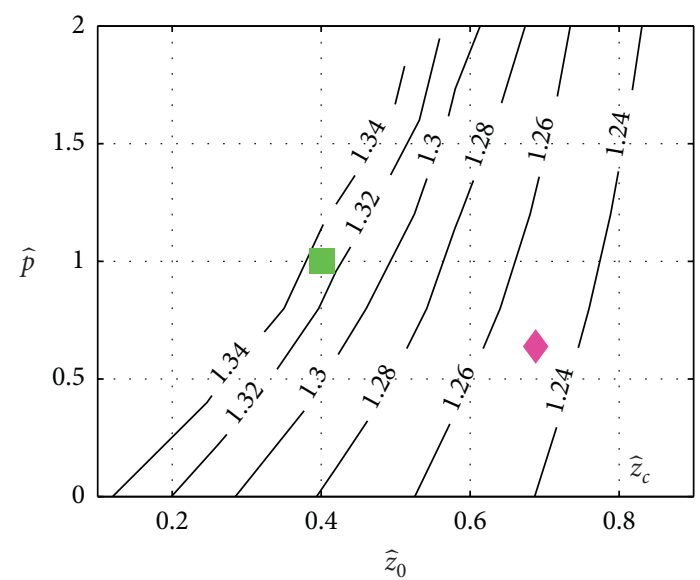

(f)

FIGURE 10: Contour plots for the design of an $X$-shaped suspension with QZS behaviour at large deflections: values of $\Omega_{C}$ and $\widehat{Z}_{C}$ on the backbone curve for different combinations of $\sigma_{1}, \widehat{z}_{0}$, and $\widehat{p}$.

system parameters indicated by the markers in Figures 5 and 6 . The FRCs of the system are solved in closed form from the amplitude-frequency equation reported in equation (13), which is quadratic in $\Omega^{2}$, and with damping ratio determined according to equation (20). The stability of the steadystate solutions is calculated by applying Floquet theory as detailed in [21] so that stable solutions are represented by solid lines and unstable solutions are represented by dashed lines in the FRCs of Figure 13. For validation purposes, numerical solutions are indicated by circles, and they are obtained by direct integration of the equation of motion of the system, where the exact expression for the static spring restoring force from equation (2) is used. In the case where a multistable solution is expected, different initial conditions were selected to validate the response on both the lower and higher amplitude branches of the frequency response curve. 


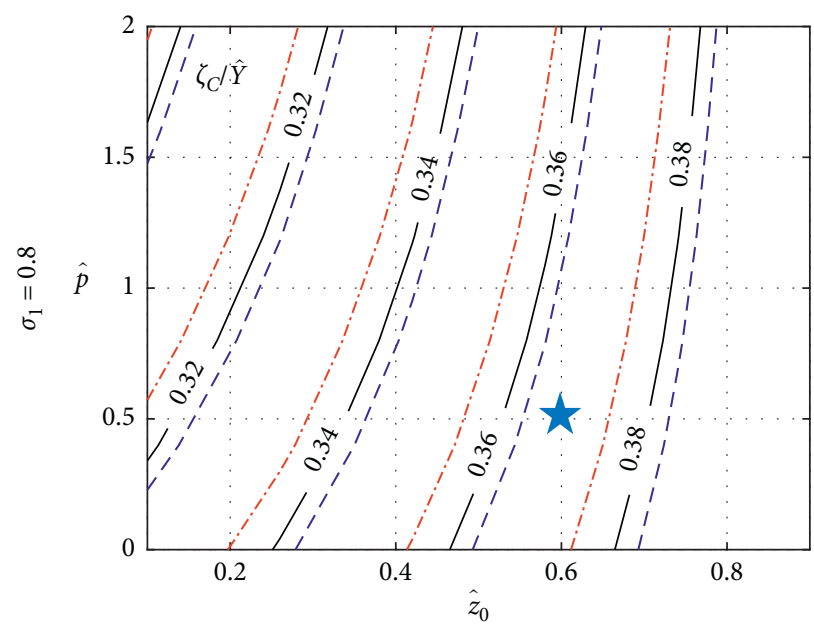

(a)

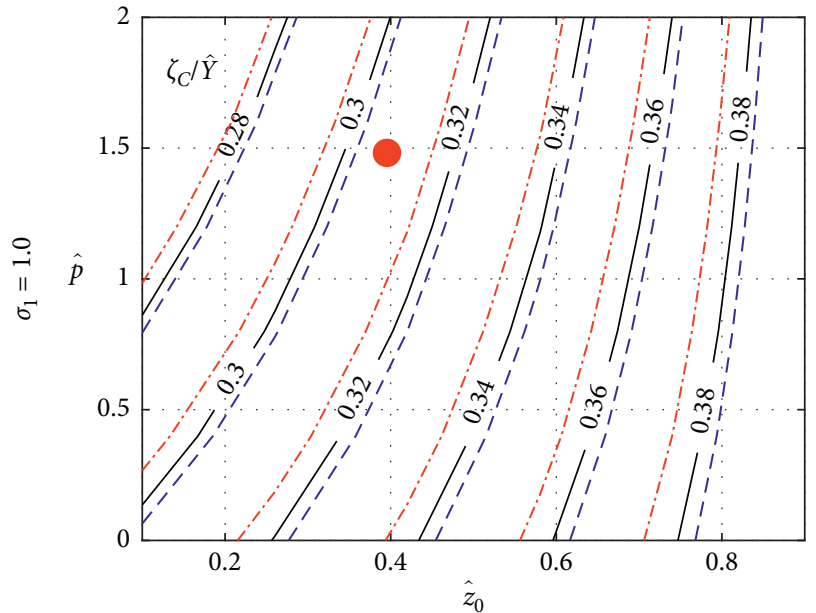

(b)

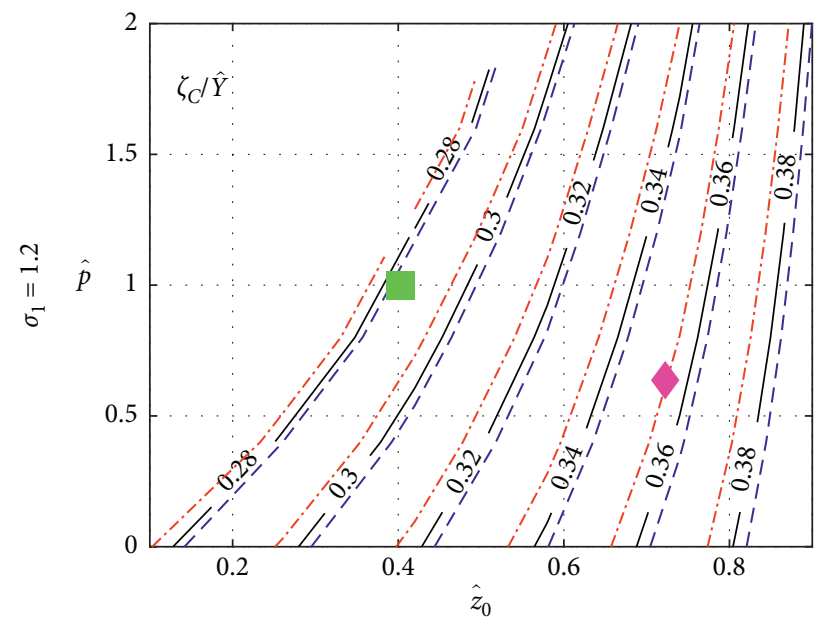

(c)

FIgURE 11: Contour plots for the design of an $X$-shaped suspension with QZS behaviour at large deflections: values of the ratio $\left(\zeta_{C} / \widehat{Y}\right)$ to attain the lowest resonance frequency, for different combinations of $\sigma_{1}, \widehat{z}_{0}$, and $\widehat{p}$, and $\widehat{Y}=0.01$ (dashed lines), $\widehat{Y}=0.3$ (solid lines), and $\widehat{Y}=0.5$ (dash-dotted lines).

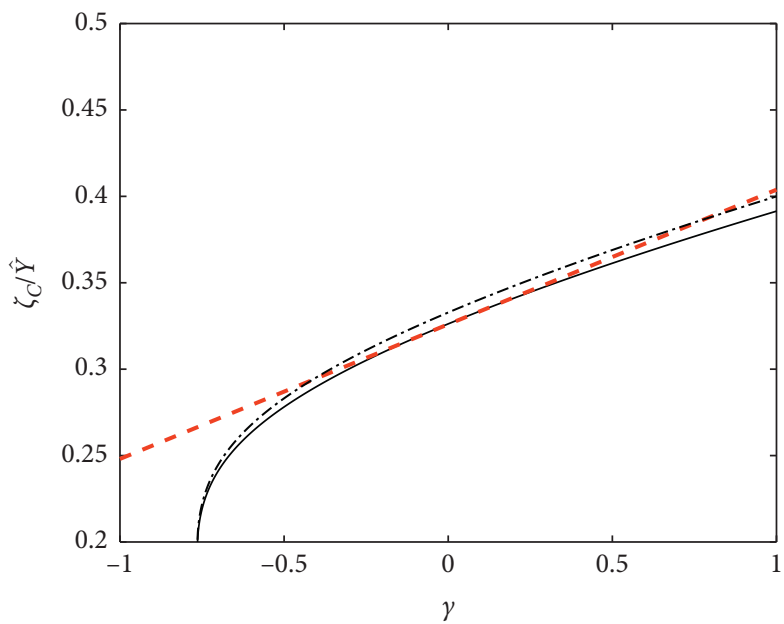

Figure 12: Ratio $\left(\zeta_{C} / \widehat{Y}\right)$ to attain the lowest resonance frequency, as a function of $\gamma$, for $\widehat{Y}=0.1$ (thin solid line) and $\widehat{Y}=0.5$ (thin dashdotted line), and approximate expression in equation (20) represented as thick dashed line. 


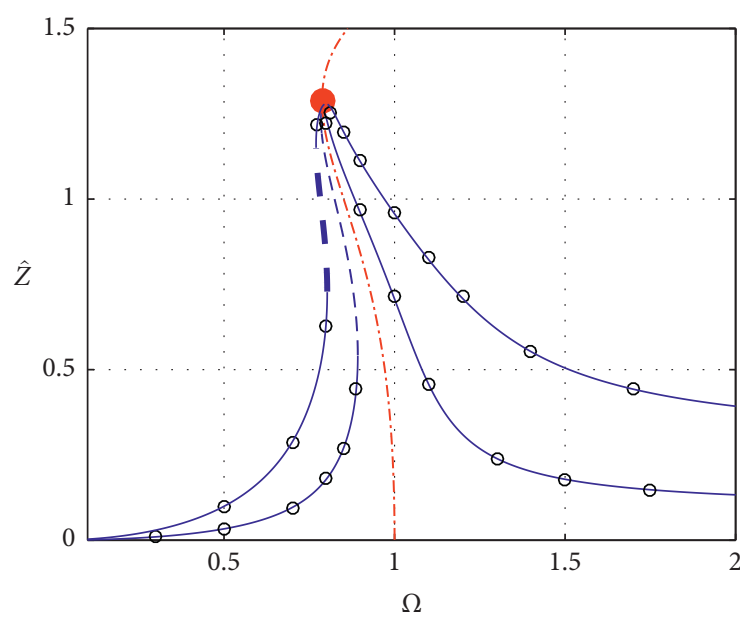

(a)

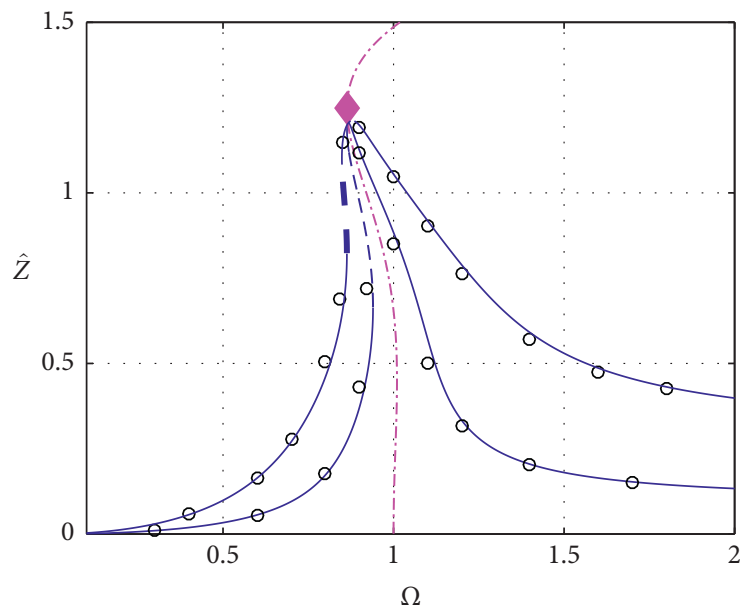

(c)

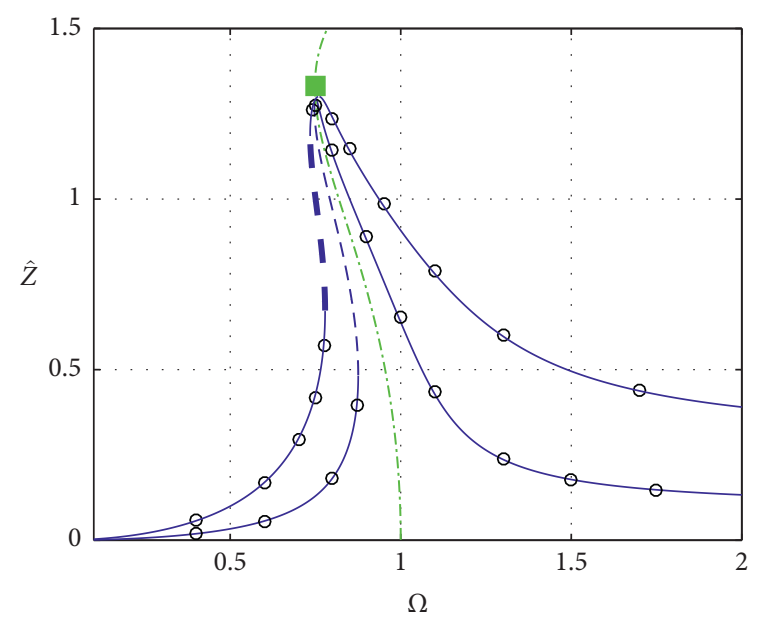

(b)

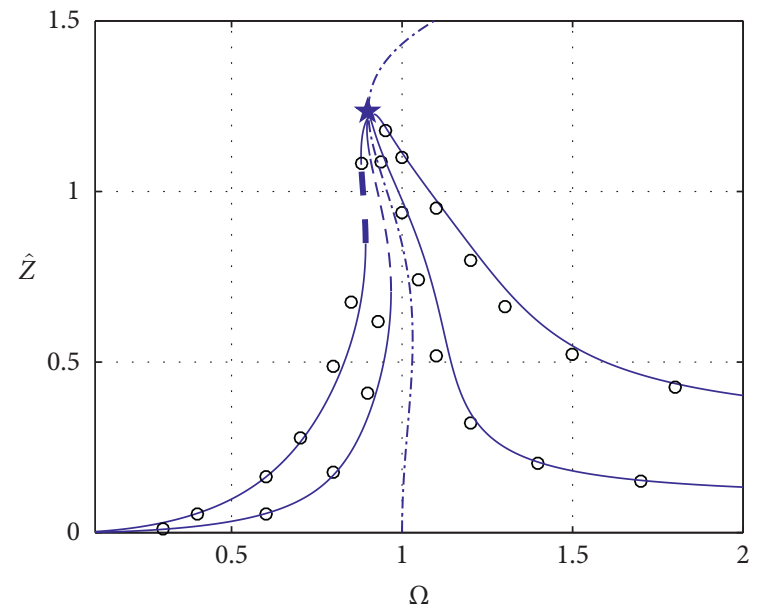

(d)

FIGURE 13: FRCs of the $X$-shaped suspension with QZS behaviour at large deflections for $\widehat{Y}=0.1$ (thin lines) and $\widehat{Y}=0.3$ (thick lines), and different values of the system parameters as indicated by the circle (a), square (b), diamond (c), and star (d) in Figures 5 and 6 . The damping is set according to equation (20). Stable analytical solutions are represented as solid lines; unstable analytical solutions are represented as dashed lines; numerical solutions are represented as empty circles; resonance peaks are represented as representative marker; and backbone curve are represented as dash-dotted line.

It can be noted that the approximate FRCs capture the dynamic behaviour of the system reasonably well. Also, it can be seen that the effect of increasing the amplitude of excitation is to widen the bandwidth around the resonance frequency, but this frequency (and its corresponding displacement amplitude) is held constant through the proper selection of the damping according to equation (20). Animation S2 in the Supplementary Material illustrates the physical assembly of the suspension system corresponding to the case in Figure 13(b), when a sinusoidal base excitation at $\Omega=1$ is provided.

\section{Conclusions}

This paper has investigated the static and dynamic characteristics of a nonlinear suspension consisting of four linear springs arranged in an $X$-shaped configuration to achieve softening characteristics and a QZS behaviour at large deflections. The analytical insight on the behaviour of the system has allowed to highlight the fundamental design strategy to achieve the desired performance. The global force-deflection curve is approximated by a seventh-order polynomial expression, and it is found that the nondimensional force-deflection characteristic with desired QZS behaviour is only dependent on the cubic stiffness coefficient, i.e., the fifth and seventh stiffness coefficients are expressed in terms of the third one. The approximate forcedeflection curve is incorporated into the equation of motion for dynamic analysis. This is performed analytically in terms of the frequency response of the system, and the effect of the parameters is studied. The investigation into the backbone curve of the harmonic response has highlighted the interesting possibility to tune the damping in the system to achieve the lowest possible resonance frequency. In particular, it is found that such damping is proportional to the excitation frequency and linearly related to the cubic 
stiffness coefficient. Numerical results have confirmed the validity of the approximate analytical formulation.

\section{Data Availability}

The data used to support the findings of this study are available from the corresponding author upon request.

\section{Conflicts of Interest}

The author declares that there are no conflicts of interest regarding the publication of this paper.

\section{Supplementary Materials}

Supplementary Materials are provided for this paper as follows. S1: animation showing the static response illustrated in Figure 7(b). S2: animation showing the dynamic response illustrated in Figure 13(b). (Supplementary Materials)

\section{References}

[1] F. Clementi, S. Lenci, and G. Rega, "1:1 internal resonance in a two d.o.f. complete system: a comprehensive analysis and its possible exploitation for design," Meccanica, vol. 55, no. 6, pp. 1309-1332, 2020.

[2] G. Gatti, M. J. Brennan, and B. Tang, "Some diverse examples of exploiting the beneficial effects of geometric stiffness nonlinearity," Mechanical Systems and Signal Processing, vol. 125, pp. 4-20, 2019.

[3] J. M. Wilson, A. Shukla, and W. Olson, "Exploiting nonlinear dynamics for design optimization of periodic response of harmonically excited, piezo-driven, buckled beams," Journal of Computational and Nonlinear Dynamics, vol. 14, Article ID 121001, 2019.

[4] Y. Chen and J. Ge, "Analysis of vibration control of nonlinear beam using a time-delayed PPF controller," Shock and Vibration, vol. 2020, Article ID 8882618, 17 pages, 2020.

[5] D. Anastasio, A. Fasana, L. Garibaldi, and S. Marchesiello, "Nonlinear dynamics of a duffing-like negative stiffness oscillator: modeling and experimental characterization," Shock and Vibration, vol. 2020, Article ID 3593018, 13 pages, 2020.

[6] Z.-Q. Lu, D. Shao, H. Ding, and L.-Q. Chen, "Power flow in a two-stage nonlinear vibration isolation system with highstatic-low-dynamic stiffness," Shock and Vibration, vol. 2018, Article ID 1697639, 13 pages, 2018.

[7] S. He, K. Chen, E. Xu, W. Wang, and Z. Jiang, "Commercial vehicle ride comfort optimization based on intelligent algorithms and nonlinear damping," Shock and Vibration, vol. 2019, Article ID 2973190, 16 pages, 2019.

[8] Q. Meng, X. Yang, W. Li, E. Lu, and L. Sheng, "Research and analysis of quasi-zero-stiffness isolator with geometric nonlinear damping," Shock and Vibration, vol. 2017, Article ID 6719054, 9 pages, 2017.

[9] M.A. Halim, K. Tao, S. Towfighian, and D. Zhu, "Vibration energy harvesting: linear, nonlinear, and rotational approaches," Shock and Vibration, vol. 2019, Article ID 5381756, 2 pages, 2019.

[10] G. Gatti, "Fundamental insight on the performance of a nonlinear tuned mass damper," Meccanica, vol. 53, no. 1-2, pp. 111-123, 2018.

[11] B. Tang and M. J. Brennan, "On the shock performance of a nonlinear vibration isolator with high-static-low-dynamic- stiffness," International Journal of Mechanical Sciences, vol. 81, pp. 207-214, 2014.

[12] Z.-Q. Lu, D.H. Gu, H. Ding, W. Lacarbonara, and L.-Q. Chen, "Nonlinear vibration isolation via a circular ring," Mechanical Systems and Signal Processing, vol. 136, Article ID 106490, 2019.

[13] G. Gatti, "A K-shaped spring configuration to boost elastic potential energy," Smart Materials and structures, vol. 28, Article ID 077002, 2019.

[14] Q. Wu, W. Zhao, W. Zhu, R. Zheng, and X. Zhao, "A tuned mass damper with nonlinear magnetic force for vibration suppression with wide frequency range of offshore platform under earthquake loads," Shock and Vibration, vol. 2018, Article ID 1505061, 18 pages, 2018.

[15] K. Guo, S. Cao, and S. Wang, "Numerical and experimental studies on nonlinear dynamics and performance of a bistable piezoelectric cantilever generator," Shock and Vibration, vol. 2015, Article ID 692731, 14 pages, 2015.

[16] G. Gatti, "Statics and dynamics of a nonlinear oscillator with quasi-zero stiffness behaviour for large deflections," Communications in Nonlinear Science and Numerical Simulation, vol. 83, Article ID 105143, 2020.

[17] F. Zhao, J. C. Ji, K. Ye, and Q. Luo, "Increase of quasi-zero stiffness region using two pairs of oblique springs," $M e$ chanical Systems and Signal Processing, vol. 144, Article ID 106975, 2020.

[18] G. Yan, H.-X. Zou, S. Wang et al., "Large stroke quasi-zero stiffness vibration isolator using three-link mechanism," Journal of Sound and Vibration, vol. 478, Article ID 115344, 2020.

[19] F. Zhao, J. Ji, Q. Luo, S. Cao, L. Chen, and W. Du, "An improved quasi-zero stiffness isolator with two pairs of oblique springs to increase isolation frequency band," Nonlinear Dynamics, vol. 104, pp. 349-365, 2021.

[20] D. Zou, G. Liu, Z. Rao, T. Tan, W. Zhang, and W.-H. Liao, "A device capable of customizing nonlinear forces for vibration energy harvesting, vibration isolation, and nonlinear energy sink," Mechanical Systems and Signal Processing, vol. 147, Article ID 107101, 2021.

[21] G. Gatti and M. J. Brennan, "Inner detached frequency response curves: an experimental study," Journal of Sound and Vibration, vol. 396, pp. 246-254, 2017. 